Rev. Int. Contam. Ambie. 37, 463-487, 2021

https://doi.org/10.20937/RICA.53865

\title{
ACTUALIZACIÓN DEL INVENTARIO NACIONAL DE EMISIONES DE 2013 PARA LA MODELACIÓN DE LA CALIDAD DEL AIRE EN EL CENTRO DE MÉXICO
}

Updating of the 2013 National Emissions Inventory for air quality modeling in Central Mexico

José Alejandro RODRÍGUEZ ZAS y José Agustín GARCÍA REYNOSO*

Instituto de Ciencias de la Atmósfera y Cambio Climático, Universidad Nacional Autónoma de México, Circuito de la Investigación Científica s/n, Ciudad Universitaria, 04510 Ciudad de México, México.

*Autor para correspondencia: agustin@atmosfera.unam.mx

(Recibido: enero 2020; accepted: septiembre 2020)

Palabras clave: contaminación atmosférica, factores de escalamiento, inventario de emisiones, modelación numérica de la atmósfera, WRF-Chem.

\section{RESUMEN}

Los inventarios de emisiones de contaminantes atmosféricos constituyen un parámetro de entrada imprescindible para los modelos de calidad del aire. Durante su proceso de elaboración, existen incertidumbres que conducen a errores en las simulaciones numéricas, siendo indiscutible la necesidad de mejorar continuamente la precisión de sus datos o ajustarlos a las condiciones reales que intenta describir. En este trabajo se aplicó el modelo de distribución espacial, temporal y de especiación química (DiETE) al Inventario Nacional de Emisiones de México de 2013 para obtener una base de datos desagregada espacial y temporalmente, lista para la modelación numérica con el modelo Weather Research and Forecasting with Chemistry (WRF-Chem) en el Centro de México. Se evaluó el desempeño del modelo en un episodio de contingencia ambiental por ozono ocurrido del 15 al 21 de mayo de 2017, a partir de la comparación con observaciones de estaciones de la Red Automática de Monitoreo Atmosférico de la Ciudad de México. Las métricas estadísticas mostraron baja correlación entre el modelo y las mediciones para todas las variables analizadas y se decidió actualizar el inventario a partir de una metodología no paramétrica de factores de escalamiento. Esto permitió obtener una mejor correlación con valores del modelo más próximos a las observaciones y que describen acertadamente el comportamiento espacial y temp,oral de las variables. Los ajustes realizados al inventario se validaron mediante su aplicación a otro caso de contingencia ambiental del 14 al 17 de marzo de 2016, apreciándose mejoras considerables en el desempeño del modelo.

Key words: atmospheric pollution, scale factors; emission inventory, atmospherical numerical modeling, WRF-Chem.

\begin{abstract}
Air pollutant emissions inventories are an essential input parameter for air quality modeling. During its elaboration process, there are uncertainties that cause errors in numerical simulations. The need to continually improve data accuracy or adjust it to the actual conditions being described is indisputable. In this work, the spatial,
\end{abstract}


temporal, and chemical speciation distribution model (DiETE) was applied to the 2013 Mexico National Emissions Inventory in order to obtain a spatially and temporarily disaggregated database, ready for numerical modeling with the Weather Research and Forecasting Model with Chemistry (WRF-Chem) in Central Mexico. Also, the model performance was evaluated for ozone in an environmental contingency episode that took place between May 15 and 21, 2017, based on the comparison with stations observations from Mexico City’s Red Automática de Monitoreo Atmosférico (automatic air quality monitoring network). Statistical metrics showed lower correlation for all variables analyzed and it was decided to update the inventory through a nonparametric methodology based on scale factors. This led us to obtain statistical parameters with larger correlations and model values closer to the observations, which accurately describe the spatial and temporal behavior of the variables. The adjustments made to the inventory were validated through its application to another case of environmental contingency from March 14-17, 2016, with considerable improvements in the performance of the model.

\section{INTRODUCCIÓN}

La contaminación del aire es un problema muy importante que enfrentan los países del mundo y un reto prioritario para la sociedad actual. México no es una excepción en este sentido. El Inventario Nacional de Emisiones para el año 2013 (INEM-2013) indica que se emitieron a la atmósfera $32.8 \mathrm{Gg}$ de contaminantes a nivel nacional, $59 \%$ provenientes de fuentes de área, $31 \%$ de fuentes móviles y $10 \%$ de fuentes fijas (INECC 2018). Existen serias afectaciones en la calidad del aire de las principales zonas metropolitanas del país, con especial atención a la Zona Metropolitana del Valle de México (ZMVM), donde los contaminantes criterio ozono $\left(\mathrm{O}_{3}\right)$ y partículas suspendidas con diámetro inferior a $10 \mu \mathrm{m}$ $\left(\mathrm{PM}_{10}\right)$ son responsables de casi la totalidad de los episodios de alta contaminación, durante los cuales se alcanzan concentraciones muy elevadas que resultan dañinas para la salud de la población (SEMARNAT 2015). Por ende, la gestión de la calidad del aire en México y específicamente en la ZMVM ha adquirido gran relevancia.

Los modelos de calidad del aire (MCA) son una poderosa herramienta para estudiar el transporte y la dinámica de los contaminantes atmosféricos, ya que simulan los procesos físicos y químicos que influyen en su comportamiento y distribución. Esto permite evaluar el estado de la calidad del aire en zonas de interés y adoptar las medidas de control necesarias para salvaguardar a la población y reducir su exposición a altas concentraciones de contaminantes (Turtós et al. 2013).

Un componente esencial de los MCA son los inventarios de emisiones (IE) de contaminantes atmosféricos, elaborados por autoridades ambientales, los cuales contienen emisiones de los principales contaminantes criterio generadas en un área (por lo general un país) provenientes de todas las fuentes (puntuales, área y móviles) y sectores (industria, transporte, comercio, servicios, hogares, vegetación, suelo) (SEMARNAT 2015). Estos IE un desempeñan decisivo en la eficiencia del proceso de simulación y la calidad de sus resultados, ya que de ellos se obtienen bases de datos de emisiones (BDE) que sirven como condiciones iniciales y de frontera para los MCA (Huang et al. 2011).

Sin embargo, en el proceso de elaboración de los IE se presenta un sinnúmero de incertidumbres (Yumimoto y Uno 2006, Lumbreras et al. 2015) que no están cuantificadas o no son reportadas por sus autores y producen errores en las simulaciones numéricas (IPCC 2000, Hao et al. 2002, Streets et al. 2003, Chang y Hanna 2004, Borrego et al. 2008, Schultz 2008, Raadgever et al. 2011, Miller et al. 2012). Por ello se requiere el desarrollo continuo de metodologías para actualizar y ajustar sus datos (Lumbreras et al. 2009, Li et al. 2010). En este sentido, Fujita et al. (1992), CARB (1997) y Funk et al. (2001) emplearon métodos de arriba hacia abajo (top-down) basados en mediciones de concentraciones de contaminantes en horarios de la mañana junto con factores de emisión; Watson et al. (2001) utilizaron técnicas de equilibrio químico de masas y especiación de compuestos orgánicos volátiles (COV) para estimar las contribuciones relativas de emisiones de diferentes fuentes; Li et al. (2010) aplicaron un método de optimización global basado en un algoritmo genético (Holland 1975, Goldberg 1989) para actualizar un IE en China para su uso en el Community Multiscale Air Quality (CMAQ) Model; y Brown (1993), Mulholland y Seinfeld 
(1995), Chang et al. (1997), Mendoza-Domínguez y Russel (2001), Mannschreck et al. (2002), Hakami et al. (2005), Gilliland et al. (2006), Yumimoto y Uno (2006), Elbern et al. (2007), Napelenok et al. (2008), Kurokawa et al. (2009), Saide et al. (2009), entre otros, emplearon diferentes técnicas de modelación inversa de la calidad del aire, comparando directamente los resultados de las simulaciones numéricas con mediciones disponibles de concentraciones de distintos contaminantes.

En el caso de México, Streit y Guzmán (1994, 1996) encontraron que las emisiones de COV y monóxido de carbono ( $\mathrm{CO}$ ) eran subestimadas en la ZMVM, y propusieron que los datos disponibles se multiplicaran por un factor de 4 para el caso de $\mathrm{COV}$ a fin de obtener resultados aceptables en la modelación de $\mathrm{O}_{3}$ con MCA. Arriaga-Colina et al. (2004) utilizaron mediciones de concentraciones matutinas de $\mathrm{COV}, \mathrm{CO}$ y óxidos de nitrógeno $\left(\mathrm{NO}_{\mathrm{x}}\right)$ en la ZMVM para obtener las razones $\mathrm{COV} / \mathrm{NO}_{\mathrm{x}}$ y $\mathrm{CO} / \mathrm{NO}_{\mathrm{x}}$, y demostraron que las reportadas en el INEM-1998 subestimaban los valores reales y debían ser incrementadas por factores de 2 y 3 , respectivamente. West et al. (2004) compararon directamente las salidas del modelo del California Institute of Technology (CIT) empleando como entrada el INEM1998, con mediciones realizadas en la campaña IMADA de 1997 para la ZMVM; concluyeron que el modelo subestimaba las concentraciones de los contaminantes analizados y propsieron incrementos a las emisiones por factores de 3 para COV y de 2 para CO. Hernández (2006) utilizó modelación inversa con el MCA Multiscale Climate Chemistry Model (MCCM) para ajustar emisiones de hidrocarburos de la ZMVM en el INEM-1998, considerando la ubicación geográfica de las fuentes. Mendoza y García (2011) también emplearon modelación inversa para escalar el IE de 1995 para la Zona Metropolitana de Guadalajara, Jalisco, México, mediante factores de ajuste, comparando las salidas del modelo CIT con mediciones. Y Trejo (2017) utilizó una metodología para escalar las emisiones del INEM-2008 y simular las concentraciones de $\mathrm{O}_{3}$ en la ZMVM con el modelo Weather Research and Forecasting Model with Chemistry (WRF-Chem), sin ajustar las emisiones de COV.

Otra limitación de los IE radica en que, generalmente, poseen una resolución espacial y temporal que impide su utilización directa en MCA, razón por la cual García-Reynoso et al. (2018) presentan y validan el modelo de distribución espacial, temporal y de especiación química (DiETE) aplicado al INEM-2008 para desagregarlo y obtener una BDE lista para su uso en MCA en México, mientras que el INECC (2017) aplica una metodología similar al INEM-2013. En ambas investigaciones se recomienda utilizar las BDE obtenidas en MCA para evaluar la calidad de sus datos en casos de estudio reales.

Teniendo en cuenta lo anterior y en una primera instancia, en este trabajo se aplica el DiETE al INEM-2013 para obtener una BDE útil para realizar simulaciones numéricas con WRF-Chem en el Centro de México, en periodos de tiempo correspondientes a contingencias ambientales por $\mathrm{O}_{3}$ ocurridas en la ZMVM. Se evalúa el desempeño del modelo a partir de una comparación con estaciones de monitoreo disponibles. Sin embargo, al presentarse una baja correlación en las métricas estadísticas calculadas, se decide actualizar el INEM-2013 a partir de una metodología no paramétrica basada en la obtención continua de factores de escalamiento, la cual se describe en la presente investigación y se aplica al área de interés, aspecto en el cual radica su novedad.

\section{MATERIALES Y MÉTODOS}

\section{Área de estudio}

El área de estudio abarca la región denominada Centro de México, que incluye los territorios pertenecientes a la Ciudad de México y los estados de México, Morelos, Hidalgo, Tlaxcala y Puebla (Fig. 1). Dentro de ella reviste especial interés la ZMVM, una

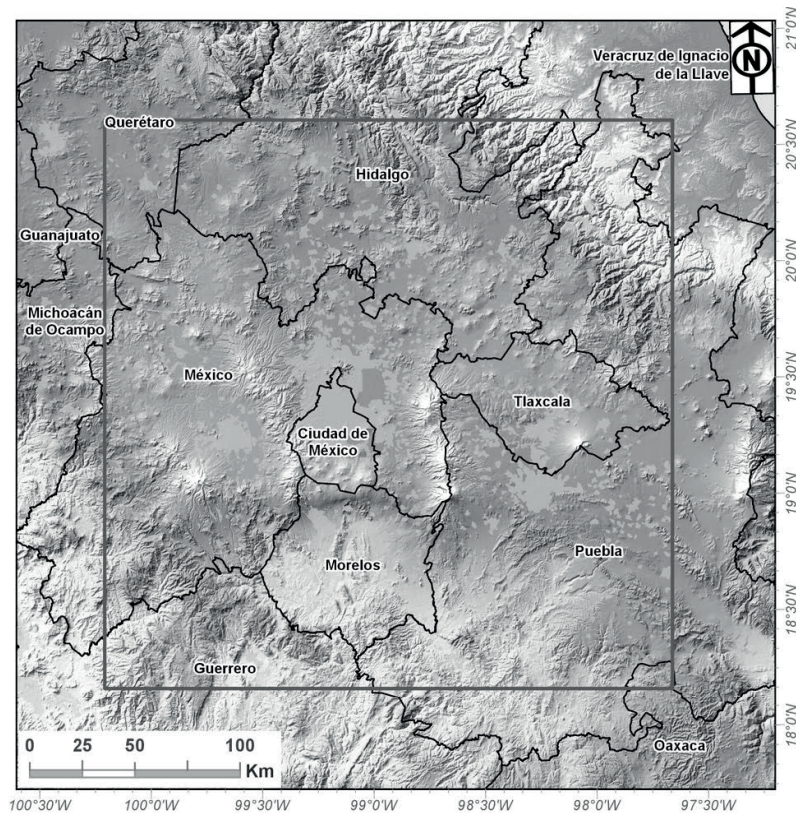

Fig. 1. Área de estudio. 
de las regiones más contaminadas del país, la cual se ubica en un valle a más de $2200 \mathrm{~m}$ de altura con factores geográficos y orográficos que dificultan la dispersión de contaminantes. Éstos son emitidos en un $30 \%$ por sus cinco millones de autos registrados, cuyas tecnologías tienen, en promedio, más de 12 años de antigüedad; además, deben considerarse las emisiones provenientes del Estado de México, que son transportadas a la ZMVM por efectos del viento. El centro geográfico de esta zona se ubica en la confluencia del paralelo $19^{\circ} 30^{\prime} \mathrm{N}$ con el meridiano $99^{\circ} 02^{\prime} \mathrm{O}$ (SEMARNAT 2002).

\section{Modelo WRF-Chem y su configuración}

En este estudio se empleó el modelo de mesoescala de nueva generación Weather Research and Forecasting with Chemistry (WRF-Chem, versión 3.9.1.1). Este modelo tiene la capacidad de simular las emisiones, transporte, mezcla y transformaciones químicas de los gases traza y aerosoles de manera acoplada con la meteorología dentro de un único sistema de coordenadas horizontales y verticales. No utiliza interpolaciones espaciotemporales y es útil para realizar estudios de calidad del aire a escala regional y global (Grell et al. 2005, Fast et al. 2006).

Para las simulaciones numéricas se construyó un dominio de modelación que cubre la región Centro de México, en el cual se ubicó una malla de $88 \times 88$ celdas con una resolución horizontal de $3 \times 3 \mathrm{~km}$ (Fig. 2), tomándose en cuenta 27 niveles verticales.

Se consideraron dos periodos de tiempo: el primero, del 15 al 21 de mayo de 2017, se utilizó para evaluar el desempeño del WRF-Chem con el IE original y posteriormente con el actualizado; el segundo,

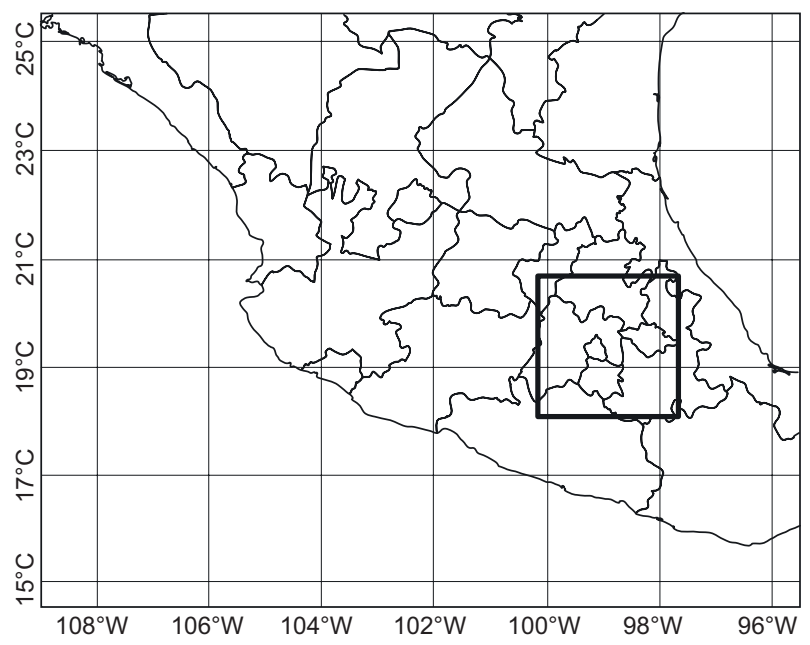

Fig. 2. Dominio de modelación. del 14 al 17 de marzo de 2016, se usó para validar la utilidad de los factores de escalamiento obtenidos, teniéndose en cuenta la activación del Programa para Contingencias Ambientales Atmosféricas (PCAA) en 2017 (Cuadro I) y 2016 (Cuadro II). En esta investigación se utiliza el tiempo en hora local.

En el primer caso se produjo un episodio de alta contaminación por $\mathrm{O}_{3}$ que comenzó el 15 de mayo de 2017 a las 16:00 horas, cuando el Índice Metropolitano de la Calidad del Aire (IMECA) registró en la estación Ajusco Medio (AJM) un valor de 151, clasificado como de muy mala calidad, alcanzándose un máximo de 186 el 20 de mayo a las 17:00 horas en AJM. Las concentraciones horarias de $\mathrm{O}_{3}$ (Fig. 3) llegaron a cifras cercanas a $180 \mathrm{ppb}$ en varias estaciones de la Red Automática de Monitoreo Atmosférico (RAMA), muy altas y dañinas para la salud, superando considerablemente la Norma Oficial Mexicana NOM-020-SSA1-2014 (SSA 2014) de 95 ppb durante cada uno de los siete días que duró la contingencia ambiental, la más prolongada desde que se aplica el PCAA (1992). Este evento estuvo relacionado con la presencia de un sistema de alta presión sobre la zona de estudio, el cual propició condiciones de estabilidad atmosférica muy favorables para la formación y acumulación de $\mathrm{O}_{3}$ y adversas para la dispersión de sus precursores, esto es, fuerte radiación solar, vientos débiles, altas temperaturas, escasas lluvias y limitado transporte de humedad (SEMARNAT 2017).

En el segundo caso el contaminante que desencadenó la contingencia ambiental también fue el $\mathrm{O}_{3}$, al alcanzarse un valor IMECA de 194 en la estación Cuajimalpa (CUA) el 14 de marzo de 2016 a las 16:00 horas, el cual llegó a un máximo de 203 una hora después en ese sitio. Las concentraciones horarias máximas de $\mathrm{O}_{3}$ alcanzaron los 203 ppb en CUA, mientras que en otras estaciones de la RAMA se observaron picos que fluctuaron entre 120 y 160 ppb (Fig. 4), condiciones que indican una muy mala calidad del aire en la ZMVM de acuerdo con el índice IMECA. Este episodio de cuatro días también estuvo relacionado con un sistema de alta presión y condiciones de estabilidad atmosférica (CAMe 2016), teniendo además la particularidad de contribuir a una actualización del PCAA, ya que se eliminó la fase de precontingencia ambiental existente hasta entonces (SEDEMA 2016).

Las condiciones meteorológicas iniciales y de frontera empleadas en WRF-Chem se generaron a partir de los datos obtenidos del Global Forecast System (GFS) desarrollado por los National Centers for Environmental Prediction (NCEP), el cual brinda información de diferentes variables con resolución espacial de $1^{\circ} \times 1^{\circ}$ y temporal de $6 \mathrm{~h}$. 
CUADRO I. ACTIVACIÓN DEL PROGRAMA PARA CONTINGENCIAS AMBIENTALES ATMOSFÉRICAS (PCAA) EN LA ZMVM EN 2017.

\begin{tabular}{lcccccc}
\hline Contaminante & $\begin{array}{c}\text { Valor } \\
\text { IMECA }\end{array}$ & $\begin{array}{c}\text { Fecha y hora } \\
\text { de activación }\end{array}$ & $\begin{array}{c}\text { Valor } \\
\text { IMECA }\end{array}$ & $\begin{array}{c}\text { Fecha y hora de la con- } \\
\text { centración máxima }\end{array}$ & $\begin{array}{c}\text { Valor } \\
\text { IMECA }\end{array}$ & $\begin{array}{c}\text { Fecha y hora } \\
\text { de desactivación }\end{array}$ \\
\hline $\mathrm{PM}_{10}$ & 153 & 6-ene (09:00) & 172 & 6-ene (11:00) & 132 & 7-ene (09:00) \\
$\mathrm{O}_{3}$ & 151 & 15 -may (16:00) & 186 & 20-may (17:00) & 104 & 21-may (19:00) \\
$\mathrm{O}_{3}$ & 162 & 22-may (15:00) & 183 & 23-may (18:00) & 105 & 24-may (18:00) \\
$\mathrm{PM}_{10}$ & 154 & 14-dic (11:00) & 162 & 14-dic (14:00) & 147 & 15-dic (09:00) \\
\hline
\end{tabular}

IMECA: Índice Metropolitano de Calidad del Aire.

CUADRO II. ACTIVACIÓN DEL PROGRAMA PARA CONTINGENCIAS AMBIENTALES ATMOSFÉRICAS (PCAA) EN LA ZMVM EN 2016.

\begin{tabular}{|c|c|c|c|c|c|c|}
\hline Contaminante & $\begin{array}{l}\text { Valor } \\
\text { IMECA }\end{array}$ & $\begin{array}{l}\text { Fecha y hora de } \\
\text { activación }\end{array}$ & $\begin{array}{l}\text { Valor } \\
\text { IMECA }\end{array}$ & $\begin{array}{l}\text { Fecha y hora de la } \\
\text { concentración máxima }\end{array}$ & $\begin{array}{l}\text { Valor } \\
\text { IMECA }\end{array}$ & $\begin{array}{l}\text { Fecha y hora de } \\
\text { desactivación }\end{array}$ \\
\hline $\mathrm{O}_{3}$ & 194 & $14-\operatorname{mar}(16: 00)$ & 203 & $14-\operatorname{mar}(17: 00)$ & 136 & $17-\operatorname{mar}(16: 00)$ \\
\hline $\mathrm{O}_{3}$ & 156 & 05 -abr $(17: 00)$ & 156 & $05-a b r(17: 00)$ & 127 & $06-a b r(17: 00)$ \\
\hline $\mathrm{O}_{3}$ & 161 & 02-may (15:00) & 192 & 04 -may (16:00) & 127 & 05 -may $(17: 00)$ \\
\hline $\mathrm{O}_{3}$ & 157 & 14 -may (17:00) & 157 & 14 -may $(17: 00)$ & 69 & 15 -may $(15: 00)$ \\
\hline $\mathrm{O}_{3}$ & 178 & 20 -may (15:00) & 188 & 20 -may (16:00) & 133 & 21-may (17:00) \\
\hline $\mathrm{O}_{3}$ & 151 & 24-may (15:00) & 151 & 24 -may (15:00) & 49 & 24-may (21:00) \\
\hline $\mathrm{O}_{3}$ & 165 & 27 -may (15:00) & 165 & 27 -may $(15: 00)$ & 109 & 28-may (18:00) \\
\hline $\mathrm{O}_{3}$ & 155 & 31-may (16:00) & 161 & 31-may (16:00) & 115 & 01 -jun (18:00) \\
\hline $\mathrm{O}_{3}$ & 152 & 08-jul (17:00) & 152 & 08-jul (17:00) & 88 & 09-jul (15:00) \\
\hline $\mathrm{O}_{3}$ & 151 & 11-ago (16:00) & 158 & 11-ago (17:00) & 127 & 12-ago $(17: 00)$ \\
\hline
\end{tabular}

IMECA: Índice Metropolitano de Calidad del Aire.
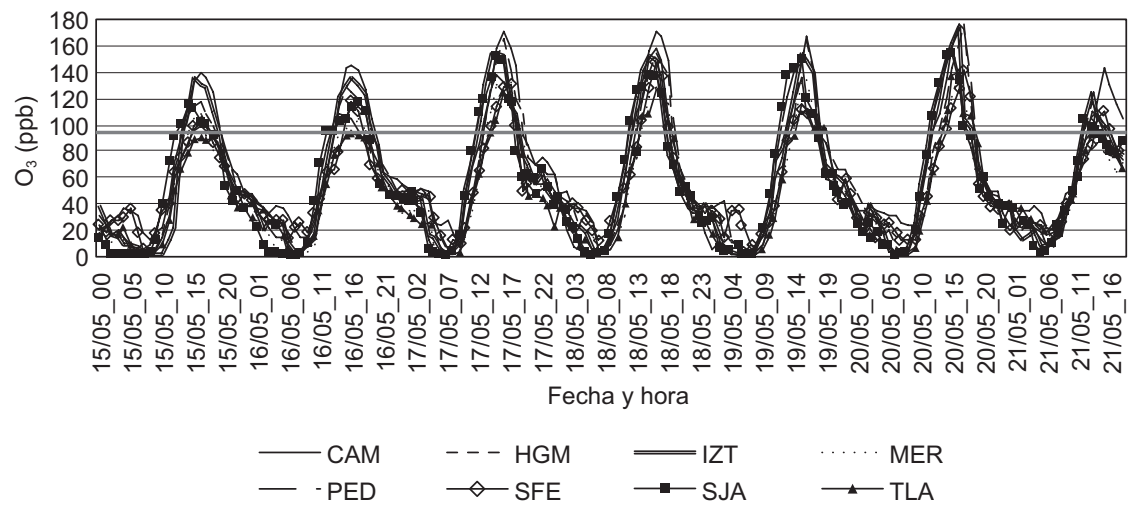

Fig. 3. Series de tiempo de las concentraciones horarias de $\mathrm{O}_{3}$ observadas en estaciones seleccionadas de la RAMA (15 al 21 de mayo de 2017). Se señala el valor de la NOM-020-SSA1-2014 (95 ppb).

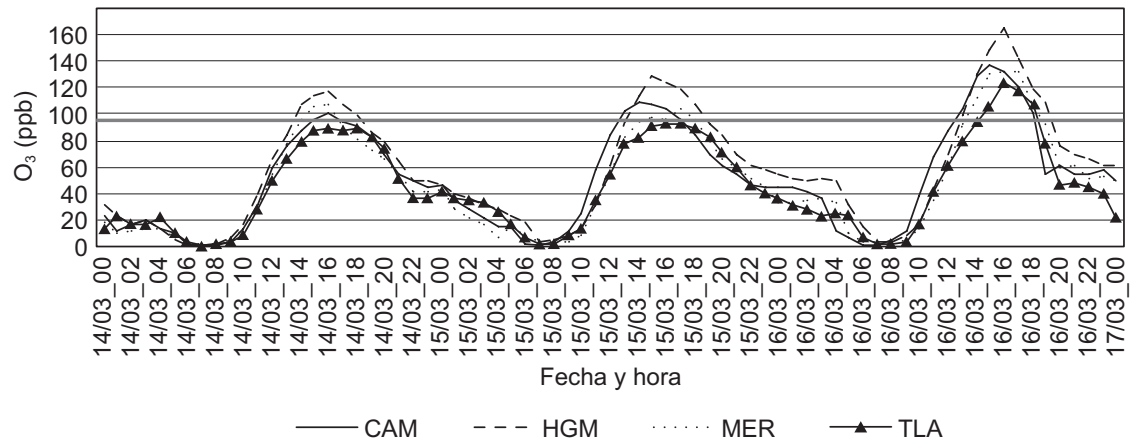

Fig. 4. Series de tiempo de las concentraciones horarias de $\mathrm{O}_{3}$ observadas en estaciones seleccionadas de la RAMA (14 al 17 de marzo de 2016). Se señala el valor de la NOM-020-SSA1-2014 (95 ppb). 
CUADRO III. PARAMETRIZACIONES EMPLEADAS EN LA MODELACIÓN CON WRF-CHEM.

\begin{tabular}{ll}
\hline Proceso & Referencia \\
\hline Capa límite planetaria & Yonsei University (YSU, Hong et al. 2006) \\
Capa superficial & MM5 Similarity (Zhang y Anthes 1982) \\
Microfísica & WRF Single-Moment class 5 (WSM5, Hong et al. 2004) \\
Uso de suelos & Noah Land-Surface Model (LSM, Chen y Dudhia 2001) \\
Radiación de onda larga & Rapid Radiative Transfer Model (RRTM, Mlawer et al. 1997) \\
Radiación de onda corta & MM5 (Dudhia 1989) \\
Cúmulos & Kain-Fritsch (Kain y Fritsch 1993, Kain 2004) \\
Mecanismo químico & Regional Acid Deposition Model v2 (RADM2, Stockwell et al. 1990) \\
Interacción Aerosoles-radiación & Activo aer_ra_feedback (Forkel et al. 2012) \\
\hline
\end{tabular}

La configuración del modelo en cuanto a parametrizaciones físicas y químicas se muestra en el cuadro III. Se destaca la utilización del mecanismo Regional Acid Deposition Model versión 2, (RADM2; Stockwell et al. 1990) para analizar la química en fase gaseosa, en el cual la distribución de emisiones por especies químicas se determina con base en la reactividad al radical hidroxilo $(\mathrm{OH})$. Considera 21 especies inorgánicas y 42 orgánicas que intervienen en 29 reacciones químicas inorgánicas (nueve fotoquímicas) y 107 orgánicas (12 fotoquímicas). Se empleó un tiempo de precalentamiento de $24 \mathrm{~h}$ y las salidas del modelo se almacenaron cada hora.

\section{Metodología para la actualización del inventario de emisiones}

En este estudio se utilizó el INEM-2013 proporcionado por la Secretaria de Medio Ambiente y Recursos Naturales (SEMARNAT), el cual cuenta con la clasificación de las emisiones de todas las fuentes fijas, móviles y de área, en sus diferentes categorías, para la ZMVM y México en general. Incluye las emisiones anuales por municipio de los principales contaminantes que afectan la calidad del aire o que son precursores de contaminantes secundarios: $\mathrm{CO}, \mathrm{NO}_{\mathrm{x}}$, dióxido de azufre $\left(\mathrm{SO}_{2}\right)$, amoniaco $\left(\mathrm{NH}_{3}\right)$, partículas suspendidas con diámetro menor a $10 \mu \mathrm{m}\left(\mathrm{PM}_{10}\right)$ y $2.5 \mu \mathrm{m}\left(\mathrm{PM}_{2.5}\right)$, y COV, entre otros (INECC 2017).

Para obtener una BDE útil en MCA, se aplicó al INEM-2013 la metodología descrita en el modelo DiETE (INECC 2017, García-Reynoso et al. 2018), en el cual la distribución espacial de emisiones en cada celda del dominio de modelación se realiza con base en datos de población, cobertura de suelo y ubicación de industrias; para fuentes móviles, se considera la fracción de la malla correspondiente a las vialidades y carreteras; para la distribución temporal se emplean los códigos fuente Source Classification Codes (SCC) de la United States Environment
Protection Agency (US-EPA), que contienen perfiles temporales mensuales, semanales y diarios por tipo de fuente.

Posteriormente se realiza la especiación de COV para cada tipo de fuente y se distribuyen las emisiones en cada perfil de compuestos químicos emitidos por proceso, lo cual requiere conocer el tipo de proceso, materias primas y condiciones de operación. Debido a que la información es limitada, se utilizan los datos SCC de la US-EPA. Finalmente, se hace la agrupación según el mecanismo químico empleado (RADM2), que asigna a cada especie química una categoría dependiendo de su reactividad con el radical $\mathrm{OH}$.

Se obtuvieron archivos diarios en formato NetCDF (Rew y Davis 1990) con información de la distribución espacial, temporal y química de las emisiones de las especies para el mecanismo RADM2, en el dominio de modelación definido. Sin embargo, al realizar simulaciones numéricas con la BDE obtenida, los resultados del modelo presentaron una baja correlación con las mediciones, como se muestra en secciones posteriores. Esto podía preverse, teniendo en cuenta que el IE corresponde a 2013 y la modelación a 2017; además, en su elaboración, se emplean factores de emisión, perfiles temporales y especiación de compuestos orgánicos que no consideran las características del área de interés, sino que provienen de estimaciones de la US-EPA. Asimismo, la incertidumbre en los datos del IE no está cuantificada ni se reporta en el mismo, por lo que se requieren acciones constantes para su actualización acordes con el caso de estudio que se desee modelar (Hao et al. 2002, Streets et al. 2003, de Foy et al. 2006, Sowden et al. 2008, Keenan et al. 2009, Li et al. 2010, Ying et al. 2012, Trejo 2017).

Teniendo en cuenta lo anterior, se llevó a cabo un proceso de actualización del IE a partir de una metodología descrita en la figura 5, la cual consiste en emplear el IE original (en este caso INEM-2013) 


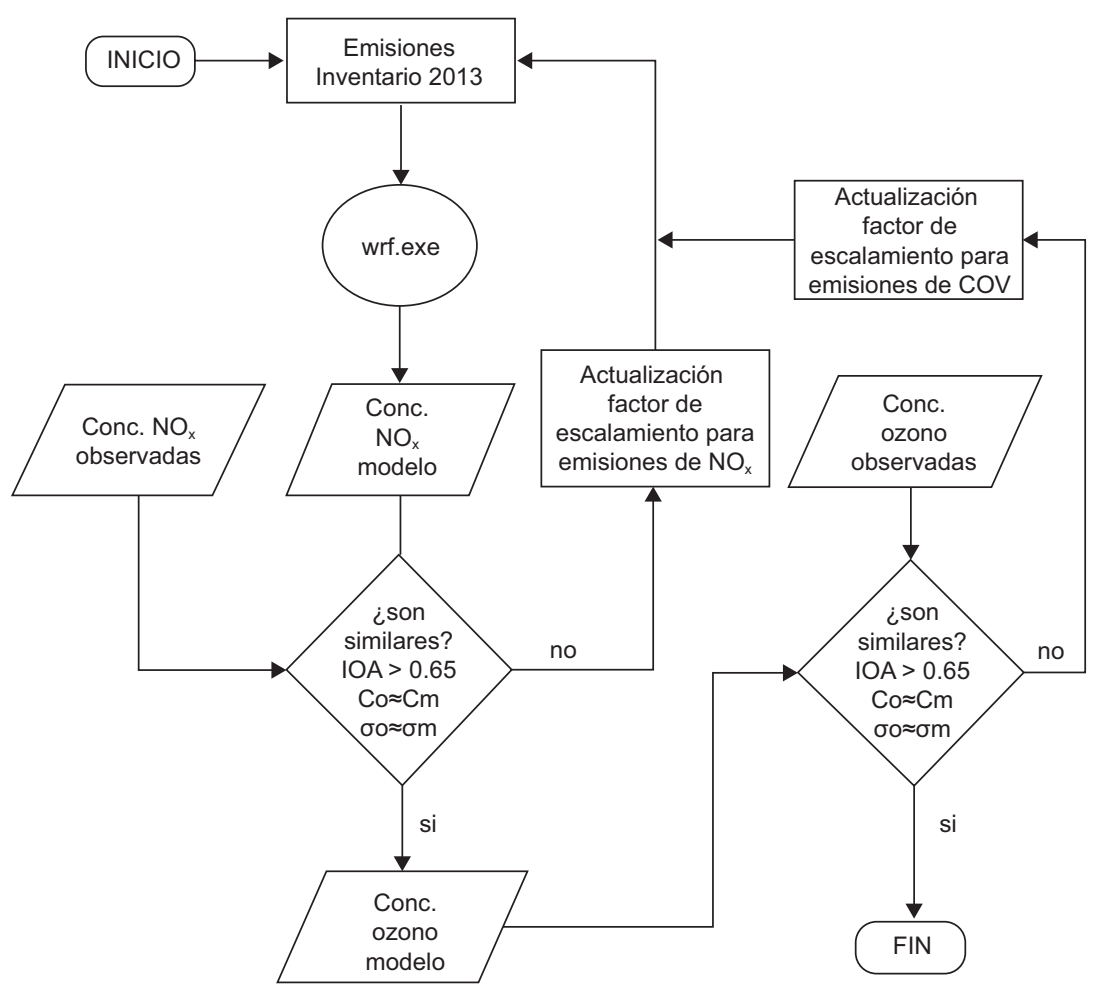

Fig. 5. Metodología de ajuste de emisiones en el INEM-2013 mediante modelación con WRF-Chem para la obtención de concentraciones de $\mathrm{O}_{3}$ similares a las observadas.

para modelar la calidad del aire y evaluar los resultados de las concentraciones obtenidas respecto a las observaciones, inicialmente para $\mathrm{NO}_{\mathrm{x}}$. Cuando las métricas estadísticas muestren valores dentro del rango de aceptable se procede a comparar los resultados para el caso del $\mathrm{O}_{3}$, con el objetivo de escalar las emisiones de COV en el IE (y las de $\mathrm{NO}_{\mathrm{x}}$, si fuera necesario) hasta que las métricas indiquen resultados aceptables.

Se realizaron simulaciones numéricas para los siguientes casos, considerando información: 1) de todas las fuentes de emisión, 2) sólo de fuentes fijas, 3) sólo de fuentes móviles y 4) sólo de fuentes de área. Luego se formó una ecuación en la que el vector de las concentraciones es igual a la matriz de datos multiplicada por el vector de los factores de escalamiento, a partir de la cual se obtiene el vector de factores de escalamiento. Este primer resultado se empleó para determinar la contribución de cada tipo de fuente a la concentración total de cada contaminante en la atmósfera y de esta forma definir un factor de escalamiento para cada uno de ellos, es decir, un valor que permita incrementar o disminuir sus respectivas emisiones en el IE original, con el fin de reducir el error.
Este primer factor de escalamiento se continúa actualizando mediante la metodología descrita en la figura 5 hasta obtener un conjunto definitivo de valores (Cuadro IV). Se observa, en general, que para las fuentes de área se propone aumentar 2.6 veces las emisiones de $\mathrm{CO}$ y 1.25 veces las de $\mathrm{SO}_{2}$, así como reducir entre el 80 y el $90 \%$ las emisiones de $\mathrm{NO}$ y $\mathrm{NO}_{2}$. Para las fuentes móviles es necesario disminuir aproximadamente a la mitad las emisiones de $\mathrm{CO}$ y $\mathrm{NO}_{2}$, así como en 15 y $90 \%$ las de $\mathrm{SO}_{2}$ y NO, respectivamente. En el caso de las fuentes puntuales, los factores de escalamiento indican que

CUADRO IV. FACTORES DE ESCALAMIENTO OBTENIDOS POR CONTAMINANTE Y TIPO DE FUENTE.

\begin{tabular}{lccc}
\hline Contaminante & Área & Móvil & Puntual \\
\hline $\mathrm{NO}$ & 0.18 & 0.10 & 1.20 \\
$\mathrm{NO}_{2}$ & 0.10 & 0.53 & 0.40 \\
$\mathrm{CO}$ & 2.60 & 0.50 & 0.50 \\
$\mathrm{SO}_{2}$ & 1.25 & 0.85 & 0.40 \\
$\mathrm{COV}$ & 2.50 & 2.50 & 2.50 \\
\hline
\end{tabular}

COV: contaminantes orgánicos volátiles. 
es preciso aumentar 1.2 veces las emisiones de $\mathrm{NO}$ y bajar entre 40 y $50 \%$ las de $\mathrm{NO}_{2}, \mathrm{CO}$ y $\mathrm{SO}_{2}$. Para todos los casos, los COV se deben incrementar por un factor de 2.5.

La aplicación de esta metodología permite obtener una BDE actualizada y útil para modelación de la calidad del aire en el área de estudio. Se estima que la calibración es eficiente, ya que los factores de escalamiento determinados a partir de las simulaciones realizadas para el caso de estudio de 2017 fueron útiles para mejorar los resultados en el caso de 2016, es decir, en diferentes periodos de tiempo y condiciones atmosféricas se obtuvo un desempeño del modelo considerablemente superior (los resultados de ambos casos se presentan en secciones posteriores). Es importante aclarar que el uso de estos factores de escalamiento para actualizar el INEM-2013 permite alcanzar resultados más próximos a los medidos respecto al original, pero a fin de obtener resultados con mayor correlación podrían variar de acuerdo con la temporada del año a modelar, la calidad de los datos meteorológicos y las modificaciones que se realicen a los datos del IE (objetivo de otra investigación en curso).

En la figura 6 se muestran, a manera de ejemplo, las distribuciones espaciales de las emisiones de $\mathrm{CO}$ y NO obtenidas a partir del INEM-2013 actualizado para el 15 de mayo de 2017 a las 03:00 y a las 18:00 horas, momentos del día en que se registran, como promedio, los valores mínimos y máximos de estas variables, debido principalmente al aumento o disminución del tráfico vehicular (Zhang et al. 2009). Las emisiones más elevadas de ambos contaminantes se observan hacia la ZMVM, en el horario de la tarde.

A modo de complemento, se presenta en la figura 7 la serie de tiempo de emisiones de $\mathrm{CO}$ y $\mathrm{NO}$ entre el 15 y el 21 de mayo de 2017, para un sitio dentro del dominio, perteneciente a la ZMVM. Se observan comportamientos característicos de las emisiones de ambos contaminantes según las horas del día (Zhang et al. 2009).

Las emisiones biogénicas se obtuvieron del modelo Model of Emissions of Gases and Aerosols from Nature (MEGAN2; Guenther et al., 1994, 2006). a)

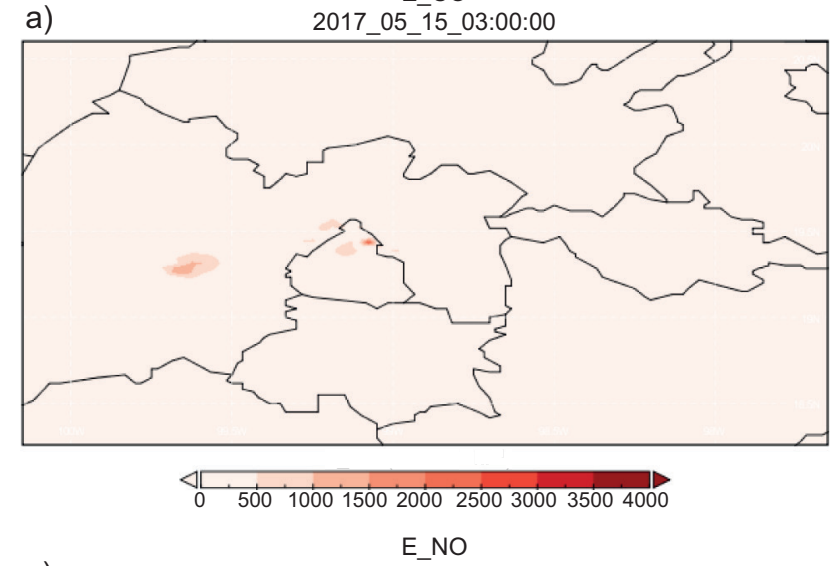

c)

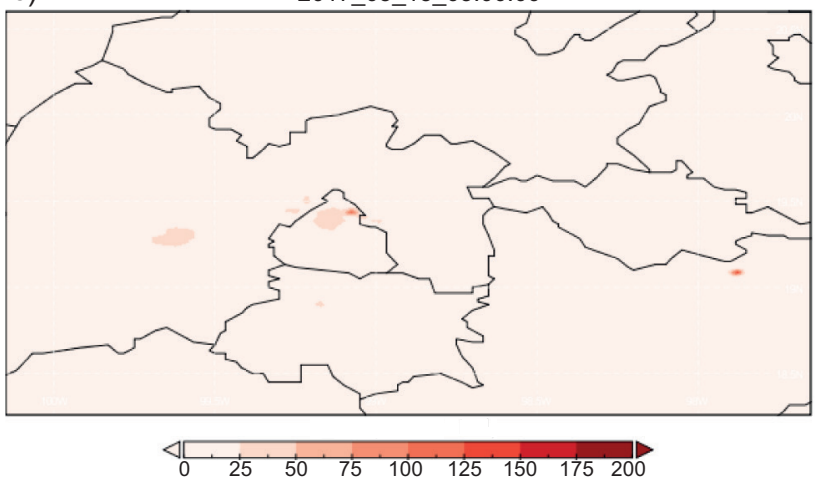

b)

E_CO

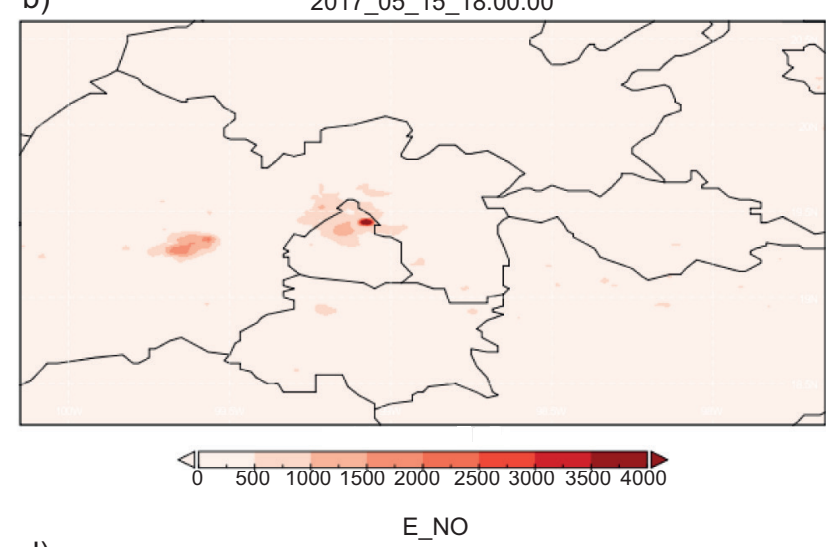

d)

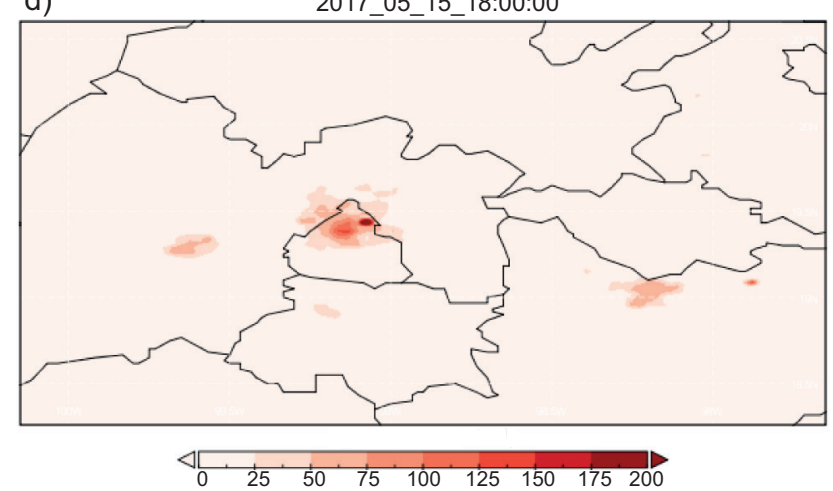

Fig. 6. Distribución espacial de las emisiones de (a y b) CO y ( c y d) NO obtenidas a partir del INEM-2013 actualizado (15 de mayo de 2017, 03:00 y 18:00 horas). 

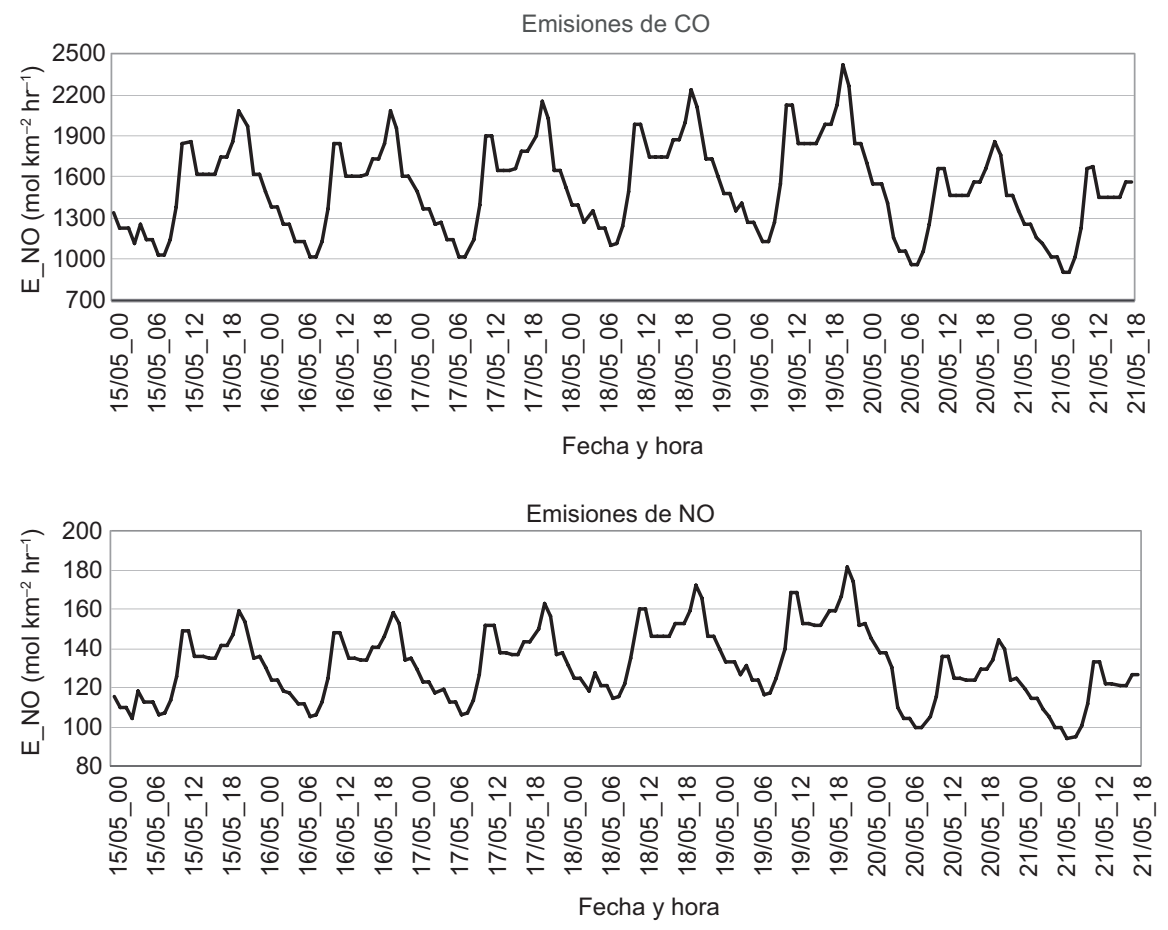

Fig. 7. Series de tiempo de las emisiones de (a) CO y (b) NO obtenidas a partir del INEM2013 actualizado (15 al 21 de mayo de 2017) para un sitio de la ZMVM.

\section{Métricas estadísticas}

Para evaluar el desempeño del WRF-Chem se compararon los valores obtenidos en las simulaciones con mediciones de estaciones de la RAMA disponibles en los periodos de tiempo seleccionados, siempre que hubieran registrado el $75 \%$ de las observaciones. De éstas se seleccionaron aquellas que tuvieran el $100 \%$ de los valores en cada caso, para que sirvieran como muestra de los análisis estadísticos, quedando las estaciones CAM (Camarones), HGM (Hospital General de México), IZT (Iztacalco), MER (Merced), PED (Pedregal), SFE (Santa Fe), SJA (San Juan de Aragón) y TLA (Tlalnepantla) para el caso de 2017 y CAM, HGM, MER y TLA para 2016. La figura 8 muestra su ubicación en el área de estudio.

Los resultados se analizaron con el Model Evaluation Tools (MET) versión 5.0, empleando la metodología de verificación celda-punto (Pielke 1984), en la cual se emparejan los valores simulados en las celdas del dominio correspondientes a la ubicación de las estaciones con los datos horarios de los contaminantes medidos. Luego se calcularon algunas métricas estadísticas (Cuadro V) muy utilizadas para evaluar cuantitativamente el desempeño del modelo y su error respecto a las observaciones. Lo más favorable sería que la media de los valores modelados $\left(\overline{C_{m}}\right)$ se aproxime a la de los valores observados $\left(\overline{C_{O}}\right)$, igual que sus

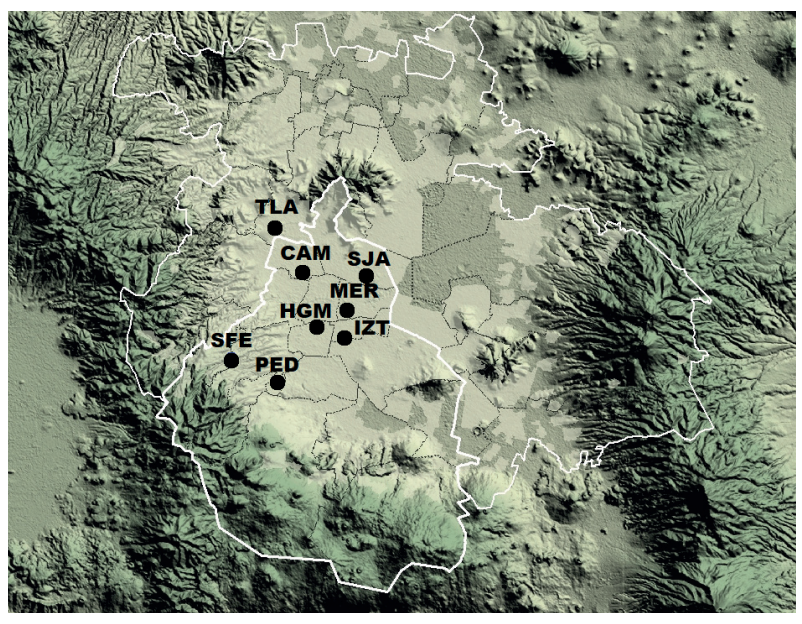

Fig. 8. Distribución de las estaciones de la RAMA consideradas en el área de estudio.

desviaciones estándar $\sigma_{o}\left(\mathrm{y} \sigma_{m}\right)$; que el sesgo medio (MB), el error medio (ME) y la raíz del error cuadrático medio (RMSE) muestren valores cercanos a 0 , en tanto que el sesgo medio normalizado (NMB) y el error medio normalizado (NME) sean cercanos a $0 \%$; y que el coeficiente de correlación $(\rho)$ y el índice de concordancia (IOA) presenten valores próximos a 1 (Pielke 1984, Willmott 1981, Willmott et al. 1985). 


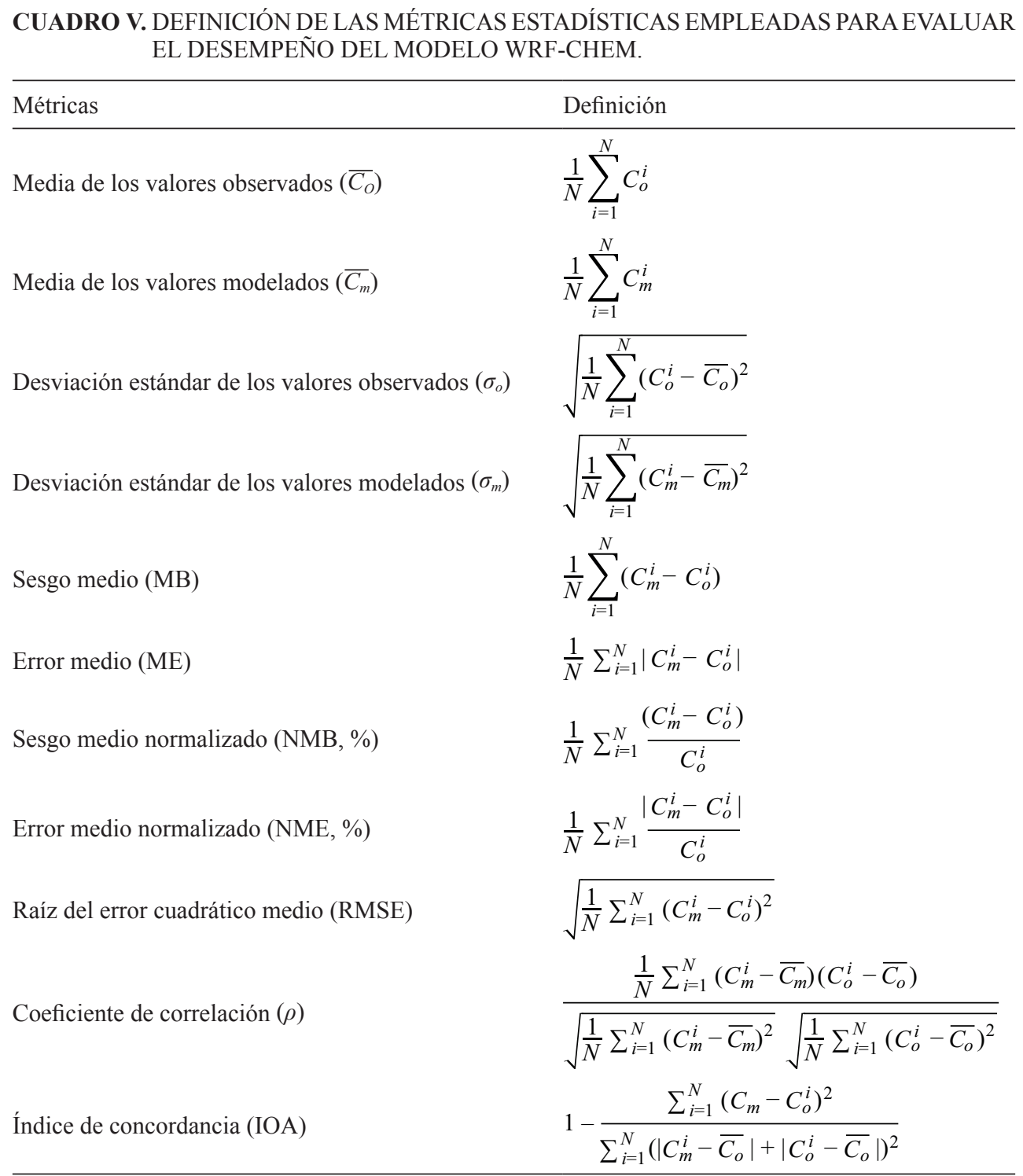

$\mathrm{N}$ : número de observaciones, $C_{m}^{i}$ : valores modelados y $C_{o}^{i}$ : valores observados.

Las variables meteorológicas analizadas en este trabajo son: temperatura superficial $\left(\mathrm{T}_{2}\right)$, humedad relativa (HR) y velocidad del viento (VV); en tanto que los contaminantes son: $\mathrm{NO}, \mathrm{NO}_{2}, \mathrm{CO}, \mathrm{SO}_{2}$, con especial énfasis en $\mathrm{O}_{3}$, principal causante de episodios de alta contaminación en el área de estudio.

\section{RESULTADOS Y DISCUSIÓN}

\section{Desempeño del modelo WRF-Chem empleando el inventario de emisiones original}

A continuación se presentan los resultados obtenidos de la modelación con WRF-Chem en el periodo del 15 de mayo de 2017 a las 00:00 horas hasta el 21 de mayo de 2017 a las 18:00 horas, a partir de la BDE inicial con la cual se contaba para las simulaciones, obtenida del INEM-2013.

En el cuadro VI aparecen valores de las métricas estadísticas calculadas para las variables meteorológicas y químicas consideradas, siendo el valor mostrado en cada caso el promedio del conjunto de todas las estaciones disponibles. Se aprecia que para el caso de $T_{2}$, HR y VV, los resultados son, en general, similares a los observados, sobre todo para $\mathrm{T}_{2}$, cuyos valores de IOA y $\rho$ son superiores a 0.90 , mientras que para HR y VV sobrepasan la aceptable cifra de 0.60 . El modelo tiende a subestimar las ob- 
CUADRO VI. MÉTRICAS ESTADÍSTICAS OBTENIDAS DE LA MODELACIÓN PARA LAS VARIABLES METEOROLÓGICAS Y CONTAMINANTES ANALIZADOS (15 AL 21 DE MAYO DE 2017) (INEM2013 ORIGINAL).

\begin{tabular}{lcccccrcc}
\hline & $\mathrm{T}_{2}$ & $\mathrm{HR}$ & $\mathrm{VV}$ & $\mathrm{O}_{3}$ & $\mathrm{NO}_{2}$ & $\mathrm{NO}$ & $\mathrm{CO}$ & $\mathrm{SO}_{2}$ \\
\hline$\overline{C_{O}}$ & 21.26 & 47.15 & 2.23 & 54.19 & 27.28 & 12.21 & 668.57 & 3.96 \\
$\overline{C_{m}}$ & 18.71 & 34.21 & 2.03 & 39.03 & 228.08 & 271.67 & 589.42 & 6.43 \\
$\sigma_{o}$ & 4.65 & 17.01 & 1.09 & 39.49 & 13.58 & 26.39 & 359.19 & 4.95 \\
$\sigma_{m}$ & 4.86 & 8.56 & 1.61 & 9.13 & 265.45 & 384.48 & 801.69 & 6.78 \\
$\mathrm{MB}$ & -2.58 & -12.96 & -0.08 & -15.17 & 200.81 & 257.79 & -81.76 & 2.47 \\
$\mathrm{ME}$ & 2.77 & 16.34 & 1.12 & 27.68 & 201.12 & 258.76 & 525.72 & 5.19 \\
$\mathrm{NMB}$ & $-1.34 \%$ & $-18.45 \%$ & $-0.81 \%$ & $-5.34 \%$ & $726.14 \%$ & $34.22 \%$ & $-14.12 \%$ & $3.45 \%$ \\
$\mathrm{NME}$ & $1.54 \%$ & $32.23 \%$ & $13.12 \%$ & $10.45 \%$ & $728.56 \%$ & $34.24 \%$ & $47.85 \%$ & $3.51 \%$ \\
$\mathrm{RMSE}$ & 3.23 & 20.34 & 1.54 & 35.89 & 328.87 & 456.89 & 773.51 & 8.66 \\
$\rho$ & 0.93 & 0.71 & 0.60 & 0.59 & 0.16 & 0.19 & 0.31 & 0.21 \\
$\mathrm{IOA}$ & 0.92 & 0.68 & 0.64 & 0.54 & 0.08 & 0.09 & 0.44 & 0.27 \\
\hline
\end{tabular}

$\overline{C_{O}}$ : media de los valores observados, $\overline{C_{m}}$ : media de los valores modelados, $\sigma_{o}$ : desviación estándar de los valores observados, $\sigma m_{o}$ : desviación estándar de los valores modelados, MB: sesgo medio, NMB: sesgo medio normalizado, $\rho$ : coeficiente de correlación, IOA: índice de concordancia, T2: temperatura superficial; HR: humedad relativa; VV: velocidad del viento.

servaciones de los parámetros meteorológicos, sobre todo para HR, cuyo MB es de -12.96 y su NMB de $-18.45 \%$. Tanto RMSE como ME para $\mathrm{T}_{2}$ y VV concuerdan con las escalas de las variables. Sin embargo, para los contaminantes analizados, el desempeño del modelo es muy deficiente. Con excepción del $\mathrm{O}_{3}$ (IOA $=0.54 \mathrm{y} \rho=0.54$, ambos por debajo de lo aceptable), en el resto de las variables IOA y $\rho$ presentan valores inferiores a 0.45 , llegando a cifras mínimas respectivas de 0.08 y 0.16 para $\mathrm{NO}_{2}$. Además, los resultados de las simulaciones muestran una sobre estimación en la concentraciones de $\mathrm{NO}_{2}$ y NO, lo que es evidente por los valores elevados de $\mathrm{MB}, \mathrm{NMB}$ y NME; estos últimos (NME) sobrepasan el $700 \%$ para $\mathrm{NO}_{2}$. En el caso del $\mathrm{CO}$, el modelo subestima las observaciones, dado por $\mathrm{MB}=-81.76 \mathrm{y}$ consolidado por $\mathrm{NMB}=-14.12 \%$. Las cifras muy altas de RMSE y ME para $\mathrm{NO}_{2}, \mathrm{NO}$ y CO indican igualmente que el error del modelo respecto a las observaciones es considerable. Por su parte, los valores de los estadísticos para $\mathrm{SO}_{2}$, con $\mathrm{RMSE}=$ $8.66, \mathrm{ME}=5.19, \mathrm{IOA}=0.27$ y $\rho=0.21$, están fuera de lo aceptable dada la escala de esta variable.

En las figuras 9, 10 y 11 se muestran, a modo de ejemplo para el caso de $\mathrm{O}_{3}$, las series temporales de las concentraciones horarias para todo el conjunto de estaciones de la RAMA consideradas y sus respectivas simulaciones numéricas (Fig. 9), las series temporales de las concentraciones horarias máximas y medias (Fig. 10) y el diagrama de dispersión para todas las concentraciones horarias (Fig. 11). Se puede apreciar que el modelo WRF-Chem no tiene un buen desempeño en la descripción del comportamiento de esta variable química en el área de estudio; es decir, aunque simula el ciclo diario de $\mathrm{O}_{3}$, no representa

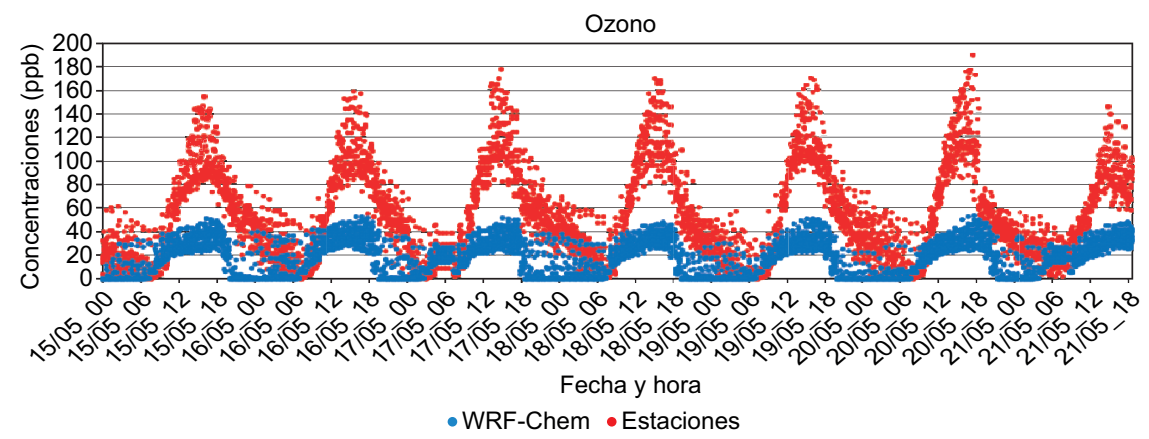

Fig. 9. Series de tiempo de todas las concentraciones horarias de $\mathrm{O}_{3}$ modeladas (INEM-2013 original) y observadas en las estaciones disponibles de la RAMA (15 al 21 de mayo de 2017). 


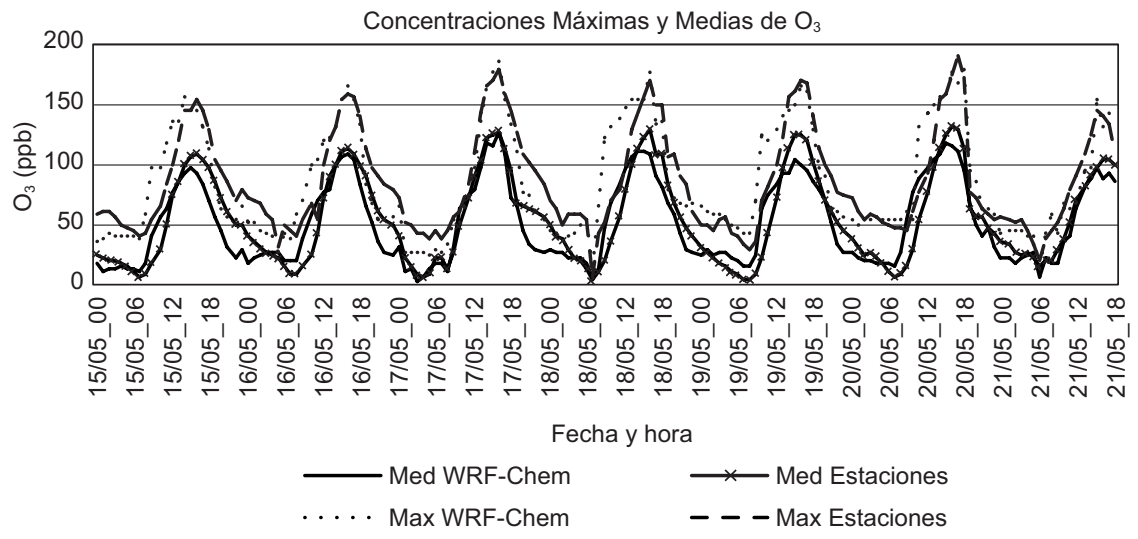

Fig. 10. Series de tiempo de las concentraciones máximas y medias horarias de $\mathrm{O}_{3}$ modeladas (INEM-2013 original) y observadas en las estaciones disponibles de la RAMA (15 al 21 de mayo de 2017).

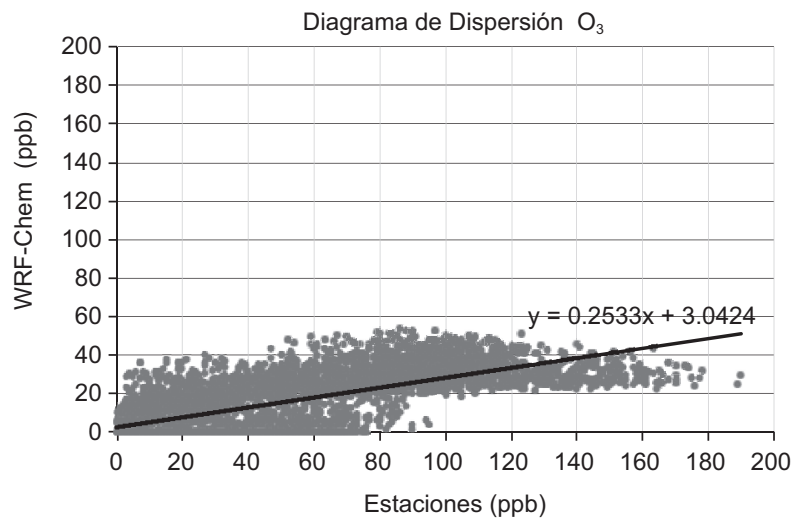

Fig. 11. Diagrama de dispersión para las concentraciones horarias de $\mathrm{O}_{3}$ modeladas (INEM-2013 original) y observadas en las estaciones disponibles de la RAMA (15 al 21 de mayo de 2017).

adecuadamente su distribución temporal en cuanto a sus valores puntuales y medios, ya que subestimando considerablemente las observaciones - sobre todo en sus picos máximos-, mostrando cifras que no sobrepasan las concentraciones de fondo.

Los resultados anteriores confirman que el INEM2013 original no es adecuado para elaborar una BDE útil para la modelación de la calidad del aire en el área de estudio. Debe destacarse que la calidad del IE es determinante para simular las concentraciones de los contaminantes atmosféricos con mejor precisión respecto a las observaciones y en menor medida influye también en el comportamiento de las variables meteorológicas, ya que el modelo WRF-Chem emplea un mecanismo que considera la interacción aerosoles-radiación (Cuadro III) para incluir el efecto de las emisiones en la meteorología; es decir, las emisiones de COV generan aerosoles orgánicos secundarios que influyen en la radiación solar que llega a la superficie, modificando la temperatura superficial y con ello otras variables meteorológicas calculadas por el modelo (Forkel et al. 2012).

\section{Desempeño del modelo WRF-Chem empleando el inventario de emisiones actualizado}

A continuación se presentan los resultados obtenidos de la modelación con WRF-Chem para el mismo periodo (15 al 21 de mayo de 2017), pero empleando la BDE actualizada que se obtuvo de aplicar los factores de escalamiento definidos en el cuadro IV al INEM-2013.

En el cuadro VII se muestran los valores de las métricas estadísticas calculadas para las variables meteorológicas y contaminantes seleccionados, apreciándose que las mismas indican una mejora cuantitativa respecto a la simulación inicial. En todos los casos los valores medios modelados son muy cercanos a los observados, con un rango de diferencia máxima del $15 \%$ para las variables meteorológicas y el $\mathrm{O}_{3}$, y algo superiores para el resto de los contaminantes, pero siempre aceptables. El modelo continúa subestimando ligeramente las observaciones en el caso de $\mathrm{T}_{2}, \mathrm{VV}$ y $\mathrm{O}_{3}$ y hasta en un $14.62 \%$ para el caso de HR (cifra que aún se considera buena), y sobrestima el resto de las variables, sobre todo $\mathrm{CO}$, que presenta un $\mathrm{MB}=61.35$, en tanto que $\mathrm{NO}_{2}$ y $\mathrm{NO}$ tienen NMB y NME superiores a $25 \%$, MB de 13.24 y 18.06, respectivamente, así como $\mathrm{ME}>25.00$, lo que las convierte en las variables con resultados más discretos, pero dentro del intervalo aceptable con valores menores al $30 \%$. Los coeficientes IOA y $\rho$ son mayores a 0.70 para todas las variables 
CUADRO VII. MÉTRICAS ESTADÍSTICAS OBTENIDAS DE LA MODELACIÓN PARA LAS VARIABLES METEOROLÓGICAS Y CONTAMINANTES ANALIZADOS (15 AL 21 DE MAYO DE 2017) (INEM-2013 ACTUALIZADO).

\begin{tabular}{lcccccccc}
\hline & $\mathrm{T}_{2}$ & $\mathrm{HR}$ & $\mathrm{VV}$ & $\mathrm{O}_{3}$ & $\mathrm{NO}_{2}$ & $\mathrm{NO}$ & $\mathrm{CO}$ & $\mathrm{SO}_{2}$ \\
\hline$\overline{C_{O}}$ & 21.26 & 47.15 & 2.23 & 54.19 & 27.28 & 12.21 & 668.57 & 3.96 \\
$\overline{C_{m}}$ & 19.06 & 41.76 & 2.14 & 51.26 & 38.76 & 28.79 & 681.42 & 5.31 \\
$\sigma_{o}$ & 4.65 & 17.01 & 1.09 & 39.49 & 13.58 & 26.39 & 359.19 & 4.95 \\
$\sigma_{m}$ & 4.73 & 12.09 & 1.46 & 26.75 & 28.13 & 34.14 & 417.06 & 5.47 \\
$\mathrm{MB}$ & -2.39 & -7.36 & -0.06 & -3.89 & 13.24 & 18.06 & 61.35 & 1.89 \\
$\mathrm{ME}$ & 2.43 & 10.94 & 1.04 & 11.65 & 27.36 & 31.67 & 72.68 & 3.66 \\
$\mathrm{NMB}$ & $-1.23 \%$ & $-14.62 \%$ & $-0.81 \%$ & $-0.34 \%$ & $25.02 \%$ & $27.19 \%$ & $13.61 \%$ & $2.49 \%$ \\
$\mathrm{NME}$ & $1.41 \%$ & $21.53 \%$ & $11.68 \%$ & $6.81 \%$ & $29.68 \%$ & $26.71 \%$ & $29.94 \%$ & $2.60 \%$ \\
$\mathrm{RMSE}$ & 3.16 & 15.08 & 1.27 & 19.36 & 31.03 & 29.52 & 36.19 & 5.17 \\
$\rho$ & 0.94 & 0.74 & 0.73 & 0.86 & 0.75 & 0.76 & 0.71 & 0.65 \\
IOA & 0.94 & 0.76 & 0.70 & 0.88 & 0.78 & 0.74 & 0.73 & 0.66 \\
\hline
\end{tabular}

$\overline{C_{O}}$ : media de los valores observados, $\overline{C_{m}}$ : media de los valores modelados, $\sigma_{o}$ : desviación estándar de los valores observados, $\sigma_{m}$ : desviación estándar de los valores modelados, MB: sesgo medio, NMB: sesgo medio normalizado, $\rho$ : coeficiente de correlación, IOA: índice de concordancia, T2: temperatura superficial; HR: humedad relativa; VV: velocidad del viento.

meteorológicas, llegando a idénticos con 0.94 en el caso de $\mathrm{T}_{2}$. Sus valores de RMSE, son muy bajos, casi insignificantes para $\mathrm{T}_{2}$ y $\mathrm{VV}$, al igual que $\mathrm{SO}_{2}$ entre los contaminantes; éstos, en general, presentan valores de IOA y $\rho$ superiores a 0.65 , destacándose el $\mathrm{O}_{3}$ con coeficientes respectivos que ascienden a 0.88 y 0.86 . En ninguno de los casos la métrica NME supera el $30 \%$, por lo que se considera que el modelo tiene muy buen rendimiento y reduce los errores respecto a las observaciones.

En la figura 12 se muestran las series de tiempo de las concentraciones horarias de $\mathrm{O}_{3}$ de todo el conjunto de estaciones consideradas con sus pares simulados correspondientes, mientras que en las figuras 13 y 14 aparecen, respectivamente, para las concentraciones medias y máximas horarias. En ambas se observa que el modelo describe la distribución temporal de la variable en términos de su ciclo diario - ampliamente estudiado en investigaciones anteriores (Tie et al. 2007, Stephens et al. 2008) y tiene la capacidad de simular tanto los valores medios como los picos diarios de concentraciones de $\mathrm{O}_{3}$ en correspondencia con las observaciones, lo cual se confirma tanto en el diagrama de dispersión de la figura 15 como en la figura 16. En esta última se muestra un resumen del porcentaje de casos observados y modelados en cada uno de los diferentes rangos definidos para las concentraciones horarias de $\mathrm{O}_{3}$.

Para analizar los resultados de manera puntual, en la figura 17 se muestran las series de tiempo de las concentraciones de $\mathrm{O}_{3}$ modeladas y observadas en algunas estaciones seleccionadas de la RAMA,

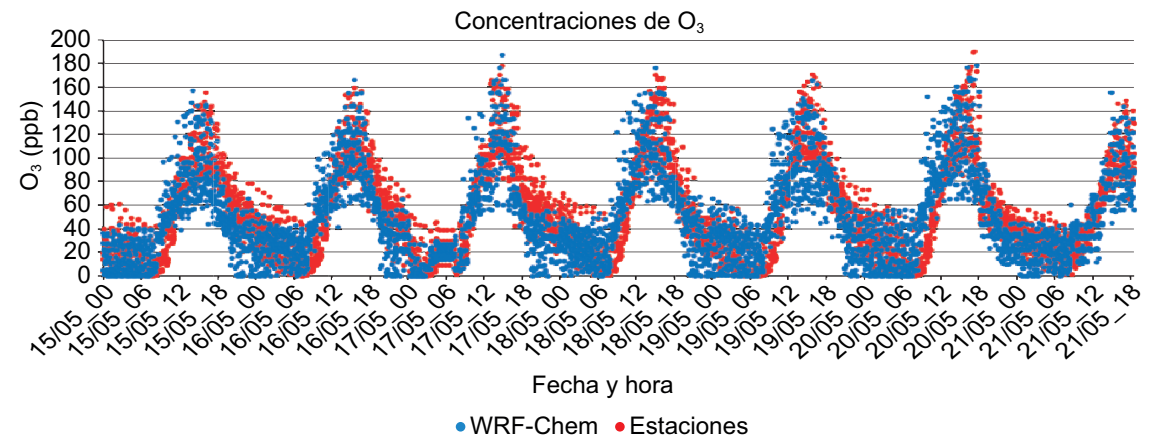

Fig. 12. Series de tiempo de todas las concentraciones horarias de $\mathrm{O}_{3}$ modeladas (INEM-2013 actualizado) y observadas en las estaciones disponibles de la RAMA (15 al 21 de mayo de 2017). 


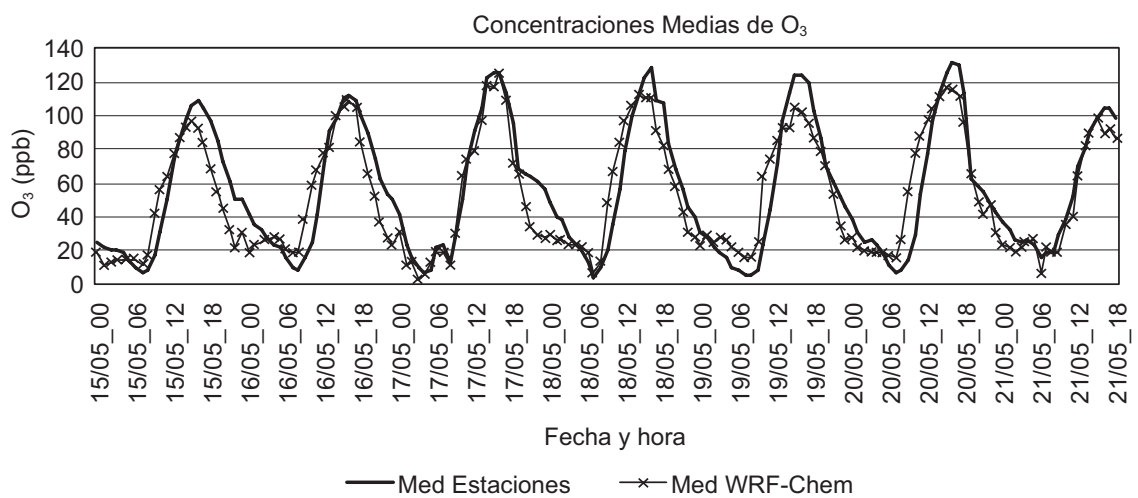

Fig. 13. Series de tiempo de las concentraciones medias horarias de $\mathrm{O}_{3}$ modeladas (INEM2013 actualizado) y observadas en las estaciones disponibles de la RAMA (15 al 21 de mayo de 2017).

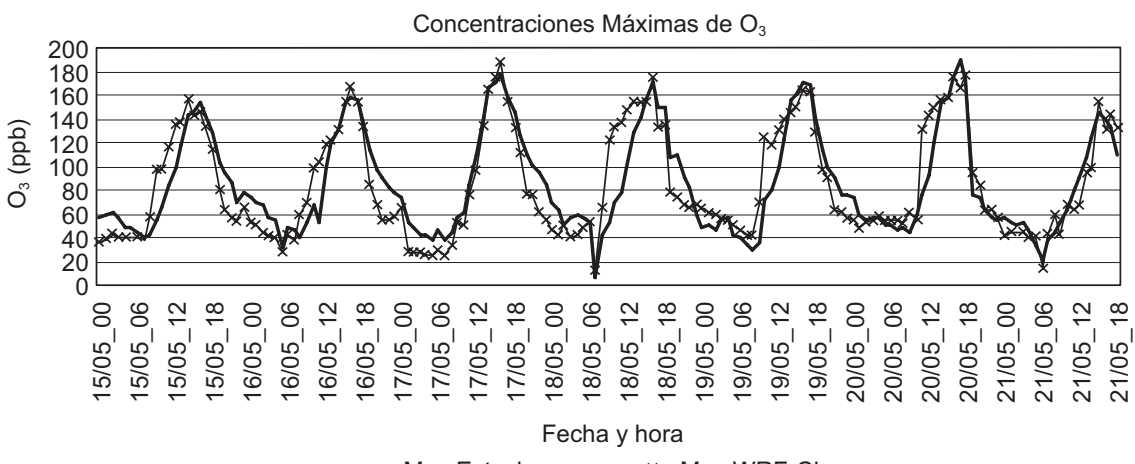

Fig. 14. Series de tiempo de las concentraciones máximas horarias de $\mathrm{O}_{3}$ modeladas (INEM2013 actualizado) y observadas en las estaciones disponibles de la RAMA (15 al 21 de mayo de 2017).

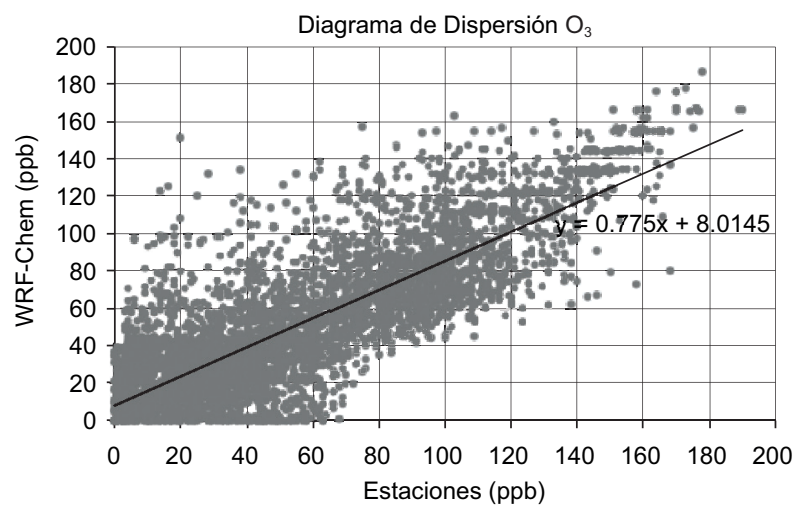

Fig. 15. Diagrama de dispersión para las concentraciones horarias de $\mathrm{O}_{3}$ modeladas (INEM-2013 actualizado) y observadas en las estaciones disponibles de la RAMA (15 al 21 de mayo de 2017).

mientras que en el cuadro VIII se presentan los valores de los estadísticos calculados. Lo más importante a señalar es que estas ocho estaciones muestran cifras de IOA y $\rho$ siempre superiores a 0.88 y muy cercanas

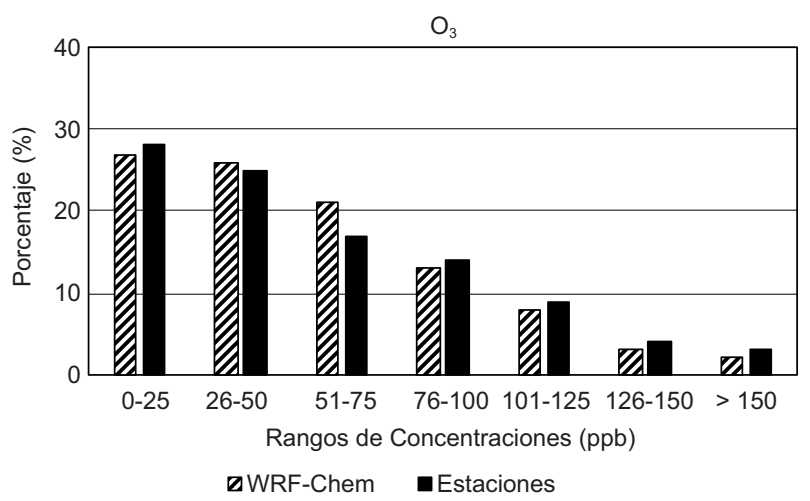

Fig. 16. Porcentaje de casos dentro de los rangos definidos de concentraciones horarias de $\mathrm{O}_{3}$ modeladas (INEM-2013 actualizado) y observadas en las estaciones disponibles de la RAMA (15 al 21 de mayo de 2017).

a 1 , con valores medios simulados que fluctúan entre 4 y $14 \%$ de las observaciones. El modelo siempre tiende a subestimar las mediciones, sobre todo los picos máximos de las concentraciones, que en el caso 

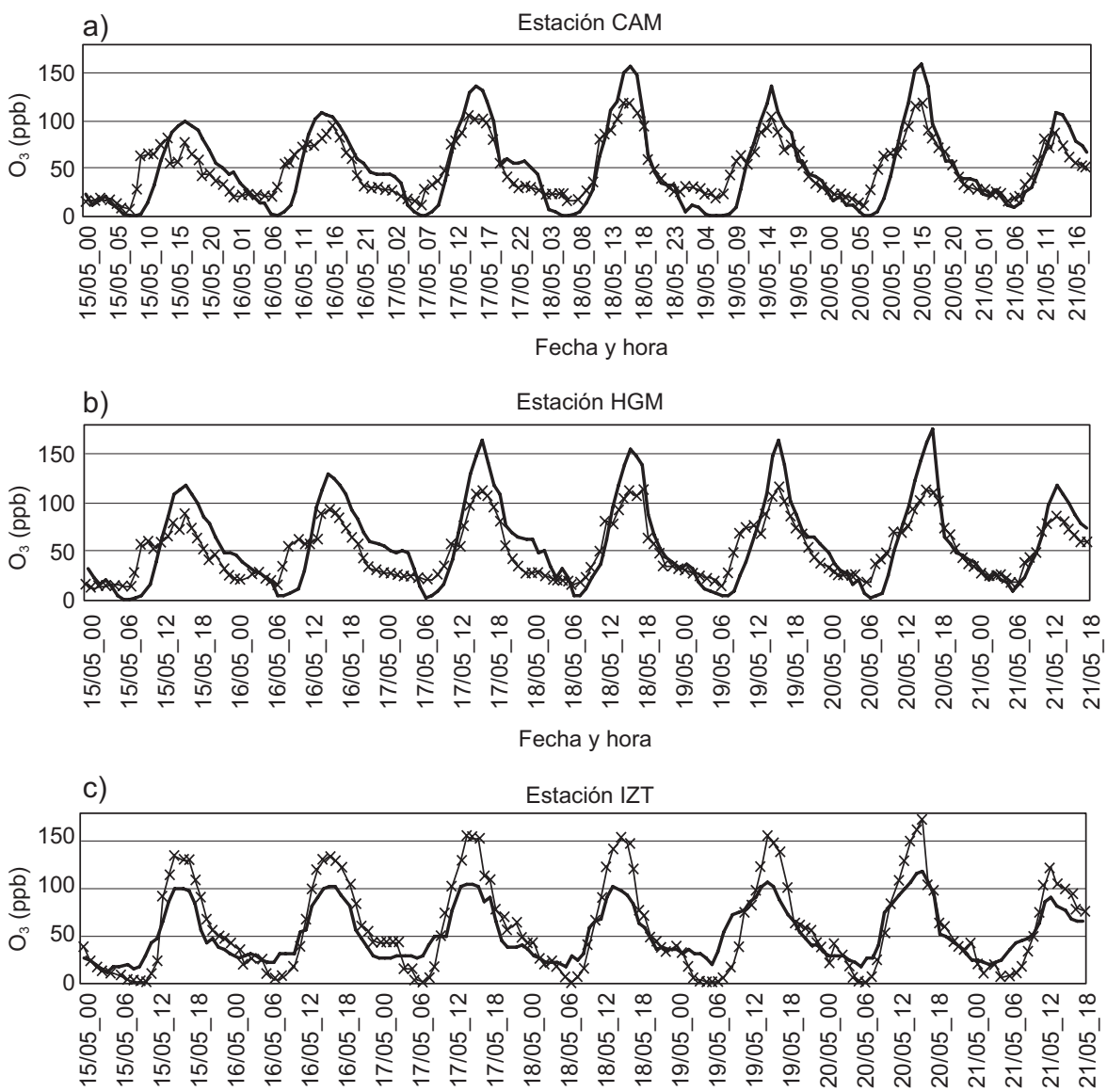

Fecha y hora

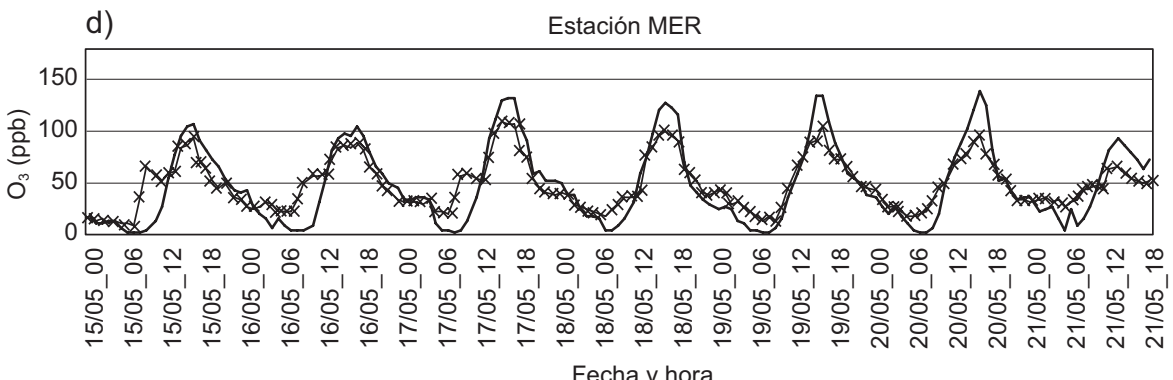

Fecha y hora

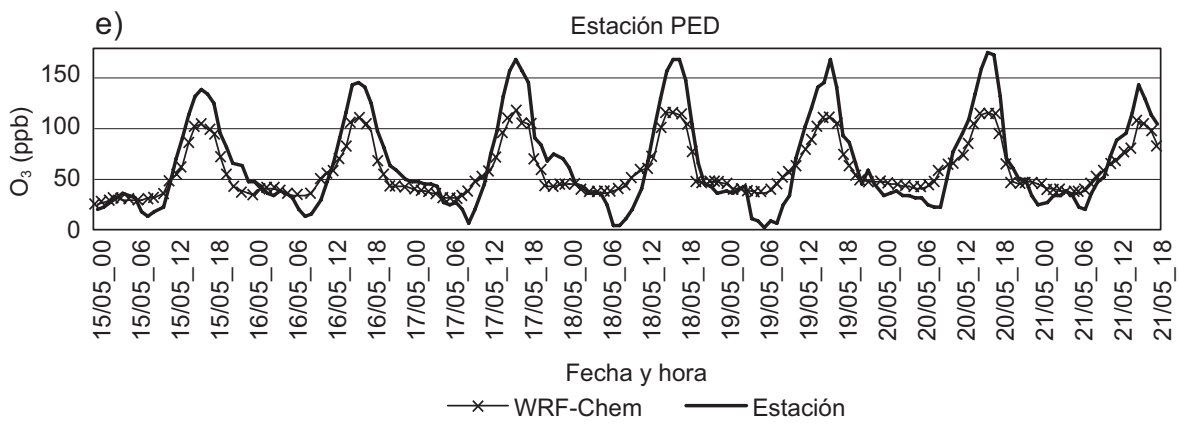

Fig. 17. Series de tiempo de las concentraciones horarias de $\mathrm{O}_{3}$ modeladas (INEM-2013 actualizado) y observadas en estaciones seleccionadas de la RAMA (15 al 21 de mayo de 2017). 

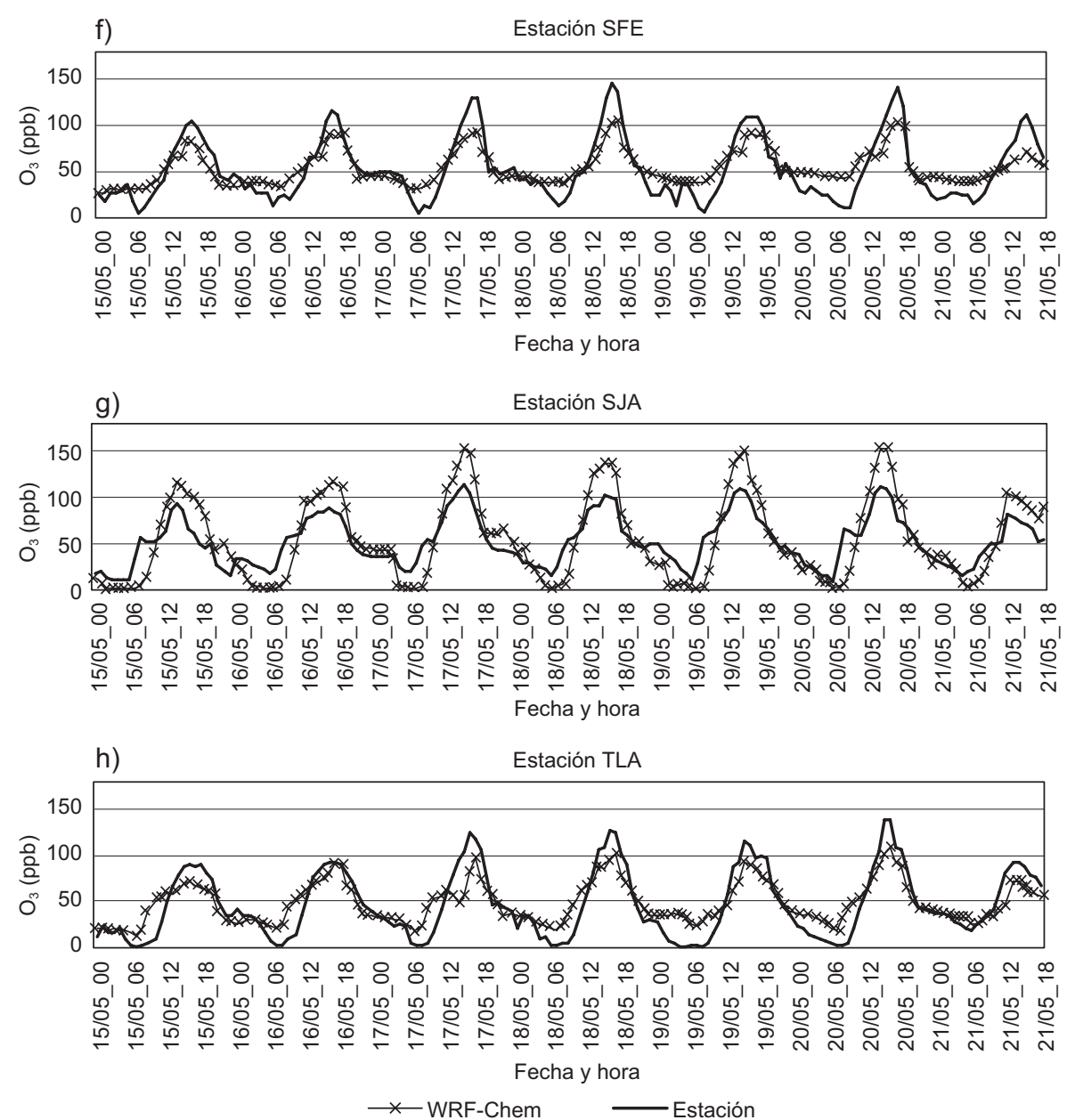

Fig. 17. Series de tiempo de las concentraciones horarias de $\mathrm{O}_{3}$ modeladas (INEM-2013 actualizado) y observadas en estaciones seleccionadas de la RAMA(15 al 21 de mayo de 2017).

CUADRO VIII. MÉTRICAS ESTADÍSTICAS OBTENIDAS DE LA MODELACIÓN DE O 3 PARA ESTACIONES SELECCIONADAS DE LA RAMA (15 AL 21 DE MAYO DE 2017) (INEM-2013 ACTUALIZADO).

\begin{tabular}{|c|c|c|c|c|c|c|c|c|}
\hline & CAM & HGM & IZT & MER & PED & SFE & SJA & TLA \\
\hline$\overline{C_{O}}$ & 51.56 & 58.83 & 58.12 & 48.79 & 66.48 & 53.67 & 54.70 & 48.39 \\
\hline$\overline{C_{m}}$ & 49.52 & 50.74 & 52.80 & 48.14 & 59.17 & 53.39 & 52.42 & 47.21 \\
\hline$\sigma_{o}$ & 41.95 & 44.28 & 46.91 & 38.40 & 46.50 & 34.23 & 44.97 & 36.84 \\
\hline$\sigma_{m}$ & 28.49 & 28.76 & 28.93 & 24.75 & 26.67 & 19.54 & 27.21 & 22.14 \\
\hline MB & -2.07 & -8.15 & -5.26 & -0.47 & -7.49 & -0.35 & -2.23 & -0.81 \\
\hline $\mathrm{ME}$ & 11.76 & 13.04 & 12.63 & 10.95 & 13.16 & 10.88 & 12.93 & 11.21 \\
\hline NMB & $-0.21 \%$ & $-0.98 \%$ & $-0.47 \%$ & $-0.09 \%$ & $-0.81 \%$ & $-0.07 \%$ & $-0.27 \%$ & $-0.13 \%$ \\
\hline NME & $7.16 \%$ & $8.34 \%$ & $7.89 \%$ & $5.74 \%$ & $8.92 \%$ & $5.62 \%$ & $8.03 \%$ & $5.41 \%$ \\
\hline RMSE & 21.41 & 23.83 & 23.07 & 18.85 & 24.07 & 18.43 & 23.21 & 18.93 \\
\hline$\rho$ & 0.89 & 0.88 & 0.92 & 0.90 & 0.92 & 0.93 & 0.91 & 0.90 \\
\hline IOA & 0.91 & 0.90 & 0.91 & 0.92 & 0.91 & 0.93 & 0.90 & 0.91 \\
\hline
\end{tabular}

media de los valores observados, media de los valores modelados, desviación estándar de los valores observados, desviación estándar de los valores modelados, MB: sesgo medio, NMB: sesgo medio normalizado, : coeficiente de correlación, IOA: índice de concordancia, CAM: Camarones, HGM: Hospital General de México, IZT: Iztacalco, MER: Merced, PED: Pedregal, SFE: Santa Fe, SJA: San Juan de Aragón, TLA: Tlalnepantla. 
$\mathrm{O}_{3}$

a) 08:00 horas 2017_05_20_08:00:00

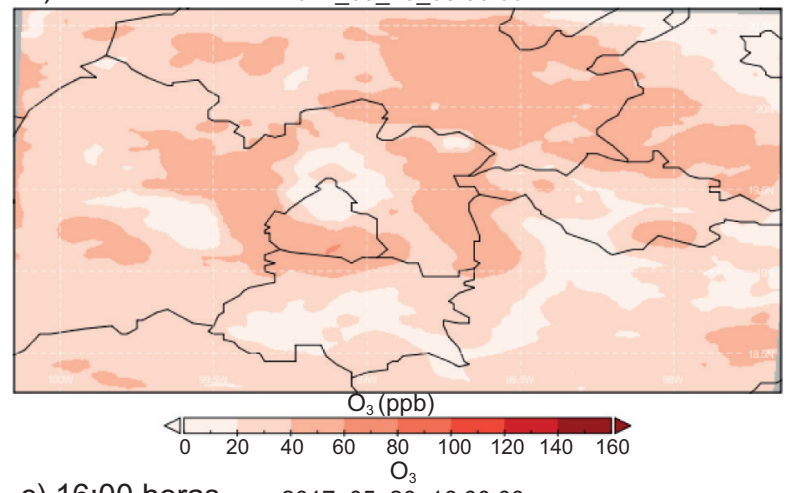

c) 16:00 horas 2017_05_20_16:00:00

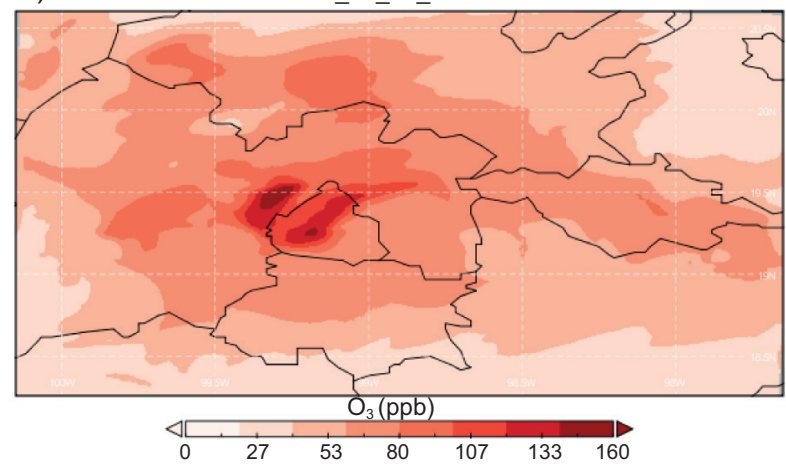

b) 13:00 horas $\quad 2017 \quad 05 \quad 20 \quad 13: 00: 00$

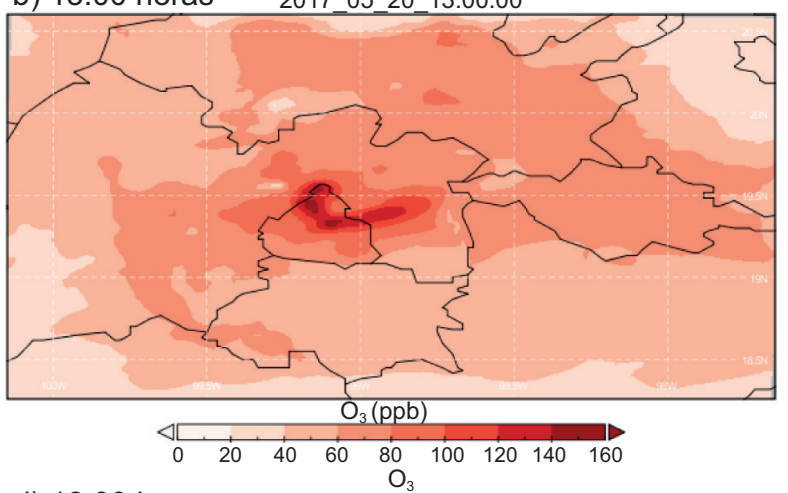

d) 18:00 horas

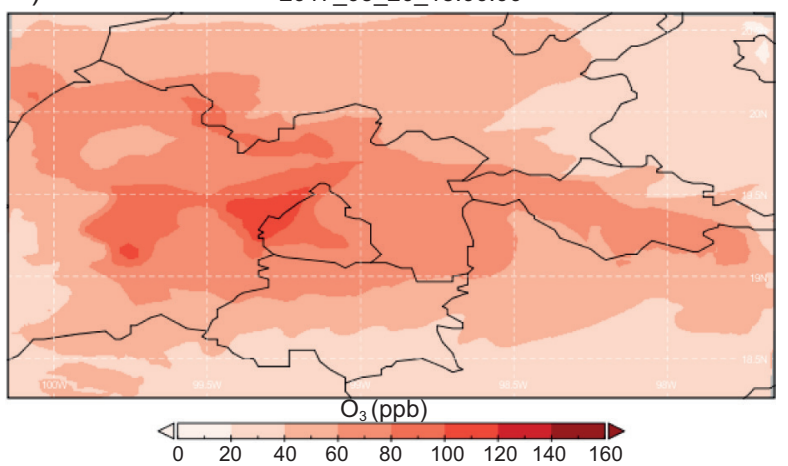

Fig. 18. Distribución espacial de las concentraciones de $\mathrm{O}_{3}$ (en ppb) obtenidas a partir del INEM-2013 actualizado (20 de mayo de 2017). (a) 08:00, (b) 13:00, (c) 16:00 y (d) 18:00 horas.

del MB varían de -0.35 (SFE) a -8.15 (HGM). El NMB se encuentra en el rango de $0 \mathrm{a}-1 \%$, en tanto que el NME siempre es inferior a $9 \%$. Por su parte, el RMSE fluctúa entre 18.43 (SFE) y 23.83 (HGM). En general, la estación SFE muestra los mejores valores de las métricas calculadas mientras que HGM los menos favorables.

Finalmente, se analiza el desempeño del modelo WRF-Chem en la simulación del comportamiento espacial de los contaminantes. En la figura 18 se muestra, a modo de ejemplo, la distribución de las concentraciones medias de $\mathrm{O}_{3}$ simuladas en el dominio de modelación a partir del INEM-2013 actualizado, para diferentes horarios del día 20 de mayo de 2017. Los resultados confirman la variación temporal de este contaminante, cuyas concentraciones medias aumentan a lo largo del día. Se comprueba que los picos máximos aparecen en zonas pertenecientes a la ZMVM o muy próximas a ésta, lo cual evidencia que es la más contaminada por $\mathrm{O}_{3}$ en el área de estudio.

Se aprecia claramente que los resultados de las simulaciones con WRF-Chem a partir del INEM-2013 actualizado son mucho más cercanos a las observaciones y con métricas estadísticas considerablemente superiores a las obtenidas para el caso original. La distribución temporal de las variables modeladas es muy similar a la observada en las estaciones disponibles, incluyendo valores medios y picos máximos con diferencias muy pequeñas. Además, el modelo es razonablemente preciso en la simulación del comportamiento del $\mathrm{O}_{3}$, variable que tiene la mayor importancia en los episodios de alta contaminación ambiental en el centro de México, específicamente en la ZMVM.

\section{Validación de los factores de escalamiento utiliza- dos para la actualización del inventario}

Con el objetivo de validar que los factores de escalamiento obtenidos sean adecuados para modificar el INEM-2013, y que la BDE derivada del mismo se pueda emplear en MCA en el centro de México en otros periodos de tiempo, se realiza una simulación de control para los días 14 al 17 de marzo de 2016, fechas en que se activó el PCAA por $\mathrm{O}_{3}$ en la ZMVM.

En el cuadro IX se muestran las métricas estadísticas obtenidas en las simulaciones con el INEM2013 actualizado para las variables meteorológicas y los contaminantes analizados, mientras que en la figura 19 aparece una comparación entre los valores 
CUADRO IX. MÉTRICAS ESTADÍSTICAS OBTENIDAS DE LA MODELACIÓN PARA LAS VARIABLES METEOROLÓGICAS Y CONTAMINANTES ANALIZADOS (14 AL 17 DE MARZO DE 2016) (INEM-2013 ACTUALIZADO).

\begin{tabular}{lcccccccc}
\hline & $\mathrm{T}_{2}$ & $\mathrm{HR}$ & $\mathrm{VV}$ & $\mathrm{O}_{3}$ & \multicolumn{1}{c}{$\mathrm{NO}_{2}$} & $\mathrm{NO}$ & $\mathrm{CO}$ & $\mathrm{SO}_{2}$ \\
\hline$\overline{C_{O}}$ & 18.83 & 38.08 & 1.82 & 48.53 & 34.81 & 21.87 & 815.83 & 3.92 \\
$\overline{C_{m}}$ & 17.28 & 33.56 & 1.57 & 46.01 & 41.76 & 30.41 & 845.62 & 4.89 \\
$\sigma_{o}$ & 5.16 & 14.92 & 1.10 & 41.13 & 18.72 & 36.93 & 414.18 & 3.87 \\
$\sigma_{m}$ & 5.59 & 11.35 & 1.36 & 37.06 & 23.09 & 42.72 & 498.55 & 4.78 \\
$\mathrm{MB}$ & -2.54 & -6.61 & -0.11 & -3.97 & 6.63 & 11.36 & 44.36 & 1.74 \\
$\mathrm{ME}$ & 2.63 & 10.26 & 1.18 & 10.29 & 24.02 & 28.03 & 77.93 & 3.38 \\
$\mathrm{NMB}$ & $-1.42 \%$ & $-12.84 \%$ & $-0.92 \%$ & $-0.48 \%$ & $21.84 \%$ & $24.39 \%$ & $16.49 \%$ & $2.13 \%$ \\
$\mathrm{NME}$ & $1.77 \%$ & $19.73 \%$ & $9.82 \%$ & $6.74 \%$ & $24.37 \%$ & $20.66 \%$ & $33.07 \%$ & $2.21 \%$ \\
$\mathrm{RMSE}$ & 3.38 & 13.73 & 1.08 & 16.74 & 28.94 & 25.22 & 39.58 & 4.52 \\
$\rho$ & 0.92 & 0.77 & 0.76 & 0.91 & 0.83 & 0.79 & 0.70 & 0.70 \\
$\mathrm{IOA}$ & 0.93 & 0.78 & 0.75 & 0.92 & 0.82 & 0.78 & 0.71 & 0.72 \\
\hline
\end{tabular}

$\overline{C_{O}}$ : media de los valores observados, $\overline{C_{m}}$ : media de los valores modelados, $\sigma_{o}$ : desviación estándar de los valores observados, $\sigma_{m}$ : desviación estándar de los valores modelados, MB: sesgo medio, NMB: sesgo medio normalizado, $\rho$ : coeficiente de correlación, IOA: índice de concordancia, T2: temperatura superficial; HR: humedad relativa; VV: velocidad del viento.

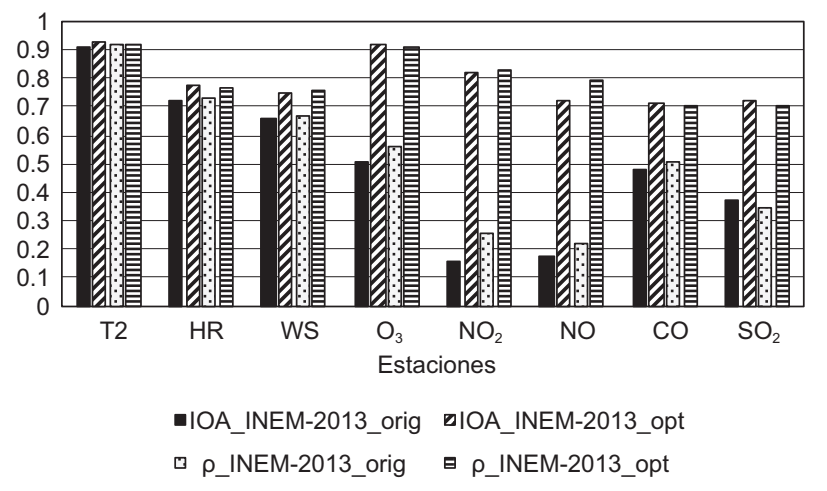

Fig. 19. Comparación de los valores de IOA y $\rho$ calculados para las distintas variables analizadas en las modelaciones a partir del INEM-2013 actualizado y original (14 al 17 de marzo de 2016).

de IOA y $\rho$ calculados para las distintas variables analizadas en las modelaciones con el IE original y actualizado. Los resultados muestran de forma general una correlación alta y los valores de IOA y $\rho$ son muy superiores en las simulaciones con INEM-2013 actualizado, principalmente para los contaminantes atmosféricos. Se destaca en este sentido el $\mathrm{O}_{3}$, cuyos valores superan 0.90 , mientras que en el caso de $\mathrm{NO}$ y $\mathrm{NO}_{2}$, inicialmente se encontraban por debajo de 0.30 y con la actualización sobrepasan 0.78 . Las variables meteorológicas muestran ligeras variaciones, pero siempre positivas, ubicándose las cifras para HR y $\mathrm{VV}$ entre 0.70 y 0.80 , mientras que para $\mathrm{T}_{2}, \mathrm{IOA}=$ 0.93 y $\rho=0.92$ debido a que el modelo considera la interacción entre la radiación y aerosoles secundarios generados por reacciones atmosféricas.
Para todas las variables meteorológicas, además de $\mathrm{O}_{3}$ y CO, los valores medios modelados son muy próximos a los observados, con menos de un $15 \%$ de variación, en tanto que para $\mathrm{NO}$ y $\mathrm{NO}_{2}$ difieren entre 17 y $20 \%$, ascendiendo hasta $30 \%$ en el caso del $\mathrm{SO}_{2}$. El WRF-Chem tiende a subestimar las mediciones de $\mathrm{T}_{2}, \mathrm{HR}, \mathrm{VV}$ y $\mathrm{O}_{3}$, con valores de MB y NMB negativos que van desde -0.11 (VV) hasta $-6.61(\mathrm{HR})$ y desde $-0.48\left(\mathrm{O}_{3}\right)$ hasta $-12.84(\mathrm{HR})$, respectivamente, en todos los casos aceptables. Asimismo, sobrestima las observaciones de $\mathrm{NO}, \mathrm{NO}_{2}, \mathrm{CO}$ y SO 2 , llegando a 44.36 en cuanto al MB para CO, y a 21.84 y $24.39 \%$ para $\mathrm{NO}_{2}$ y NO, respectivamente, en el caso del NMB. De igual forma, se aprecian cifras muy bajas de RMSE en los casos de VV (1.08), $\mathrm{T}_{2}$ (3.38) y $\mathrm{SO}_{2}$ (4.52), más altas para $\mathrm{NO}$ (25.22), $\mathrm{NO}_{2}$ (28.94) y $\mathrm{CO}(39.58)$, mientras que NME siempre es inferior al $25 \%$, excepto para $\mathrm{CO}(33.07 \%)$.

Debe señalarse que, en este caso de estudio de 2016, además de evidenciarse la superioridad del INEM-2013 actualizado respecto al original, se aprecia que las métricas estadísticas calculadas para el conjunto de todas las estaciones analizadas son incluso superiores a las obtenidas para el periodo de 2017, es decir, hay una mayor correspondencia entre la modelación y las observaciones.

En la figura 20 se muestra la serie de tiempo de las concentraciones horarias de $\mathrm{O}_{3}$ de todo el conjunto de estaciones disponibles con sus pares simulados a partir del INEM-2013 actualizado. Se confirma que el WRF-Chem tiene la capacidad de describir el ciclo diario del $\mathrm{O}_{3}$, sobre todo en los valores medios, lo cual se puede comprobar en el diagrama de dispersión 


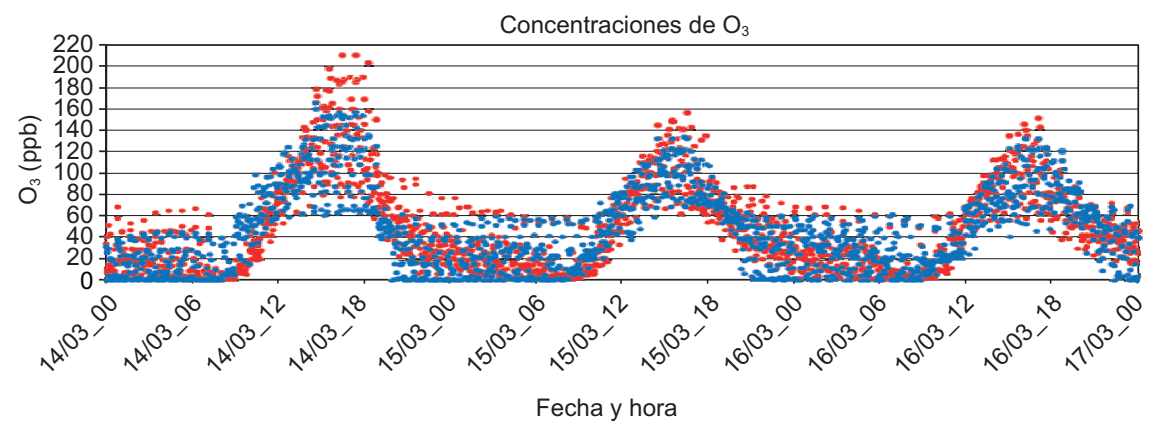

Fig. 20. Series de tiempo de todas las concentraciones horarias de $\mathrm{O}_{3}$ modeladas (INEM-2013 actualizado) y observadas en las estaciones disponibles de la RAMA (14 al 17 de marzo de 2016).

de la figura 21. Sin embargo, en cuanto a los picos de concentraciones máximas, el modelo los detecta en el horario adecuado, pero se aprecian algunas diferencias respecto a los valores observados, los cuales

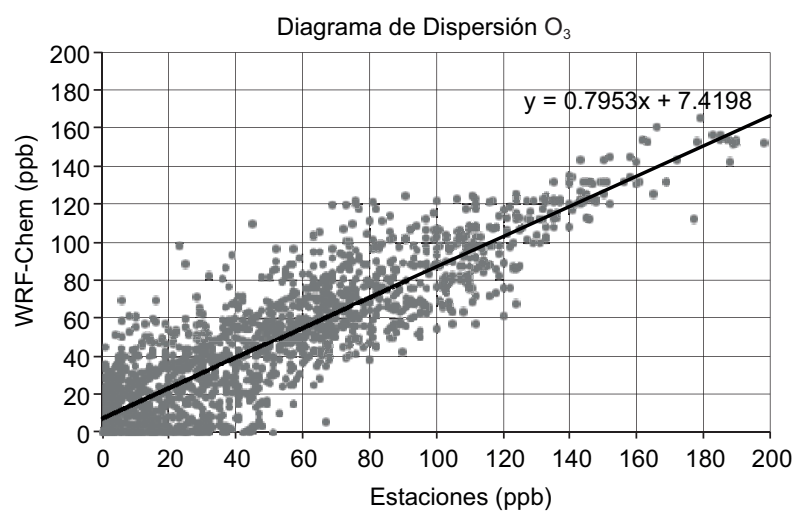

Fig. 21. Diagrama de dispersión de las concentraciones horarias de $\mathrm{O}_{3}$ modeladas (INEM-2013 actualizado) y observadas en las estaciones disponibles de la RAMA (14 al 17 de marzo de 2016). se subestiman, sobre todo para el día 14 cuando los máximos sobrepasaron los 175 e incluso los 200 ppb, mientras que las simulaciones apenas reflejaron cifras ligeramente superiores a $150 \mathrm{ppb}$. Esta situación puede deberse a la influencia en las concentraciones de $\mathrm{O}_{3}$ del transporte de contaminantes de regiones cercanas hacia el área de estudio, pero que no puede cuantificarse en la modelación porque esas emisiones no están consideradas dentro del dominio de la BDE que se obtiene del INEM-2013.

Las series de tiempo de las concentraciones horarias de $\mathrm{O}_{3}$ modeladas y observadas en estaciones seleccionadas de la RAMA se presentan en la figura 22, mientras que en el cuadro $\mathbf{X}$ aparecen los valores de las métricas estadísticas calculadas. Las cifras de IOA y $\rho$ indican alta correlación en todos los casos, siempre superior a 0.89 . Los valores medios simulados y observados difieren en un $10 \%$ para CAM y en alrededor de un $20 \%$ para el resto de las estaciones. El modelo tiende a subestimar las mediciones, sobre todo con picos máximos en el MB, el cual fluctúa entre -3.73 (CAM) y -11.79 (HGM), en tanto que

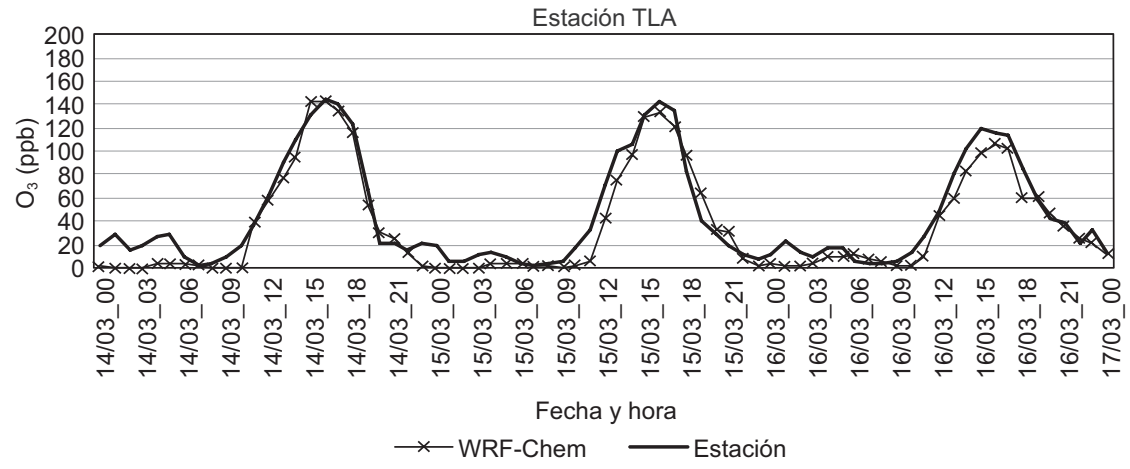

Fig. 22. Series de tiempo de las concentraciones horarias de $\mathrm{O}_{3}$ modeladas (INEM-2013 actualizado) y observadas en estaciones seleccionadas de la RAMA (14 al 17 de marzo de 2016). 
CUADRO X. MÉTRICAS ESTADÍSTICAS OBTENIDAS DE LA MODELACIÓN DE O3 PARA ESTACIONES SELECCIONADAS DE LA RAMA (14 AL 17 DE MARZO DE 2017) (INEM-2013 ACTUALIZADO).

\begin{tabular}{lcccc}
\hline & CAM & HGM & MER & TLA \\
\hline$\overline{C_{O}}$ & 43.12 & 48.21 & 37.04 & 43.67 \\
$\overline{C_{m}}$ & 39.35 & 38.41 & 29.51 & 35.82 \\
$\sigma_{o}$ & 46.08 & 49.54 & 39.86 & 44.51 \\
$\sigma_{m}$ & 44.89 & 48.67 & 38.41 & 44.32 \\
MB & -3.73 & -11.79 & -8.52 & -7.83 \\
ME & 11.23 & 13.28 & 8.69 & 8.40 \\
NMB & $-0.34 \%$ & $-1.43 \%$ & $-0.96 \%$ & $-0.86 \%$ \\
NME & $7.09 \%$ & $7.77 \%$ & $5.12 \%$ & $5.01 \%$ \\
RMSE & 20.24 & 23.16 & 15.08 & 13.21 \\
$\rho$ & 0.89 & 0.90 & 0.92 & 0.93 \\
IOA & 0.90 & 0.92 & 0.92 & 0.94 \\
\hline
\end{tabular}

$\overline{C_{O}}$ : media de los valores observados, $\overline{C_{m}}$ : media de los valores modelados, $\sigma_{o}$ : desviación estándar de los valores observados, $\sigma_{m}$ : desviación estándar de los valores modelados, MB: sesgo medio, NMB: sesgo medio normalizado, $\rho$ : coeficiente de correlación, IOA: índice de concordancia, CAM: Camarones, HGM: Hospital General de México, MER: Merced, TLA: Tlalnepantla

$\mathrm{O}_{3}$

a) 08:00 horas 2016_03_15_08:00:00

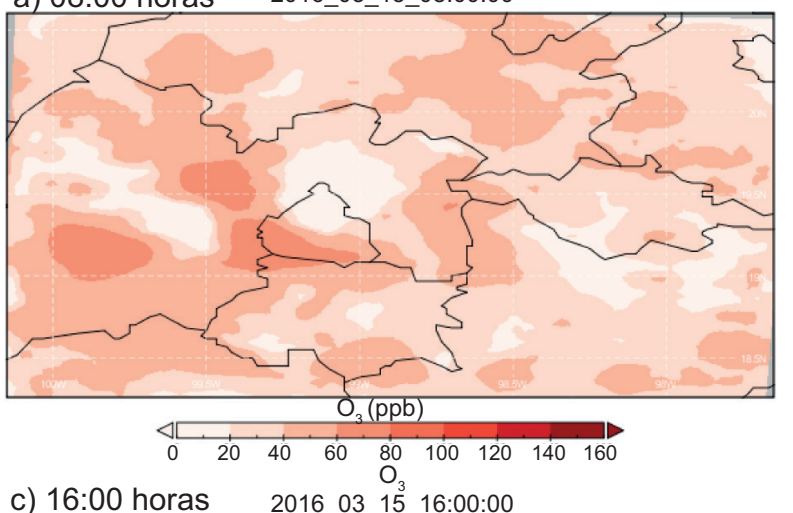

c) 16:00 horas 2016_03_15_16:00:00

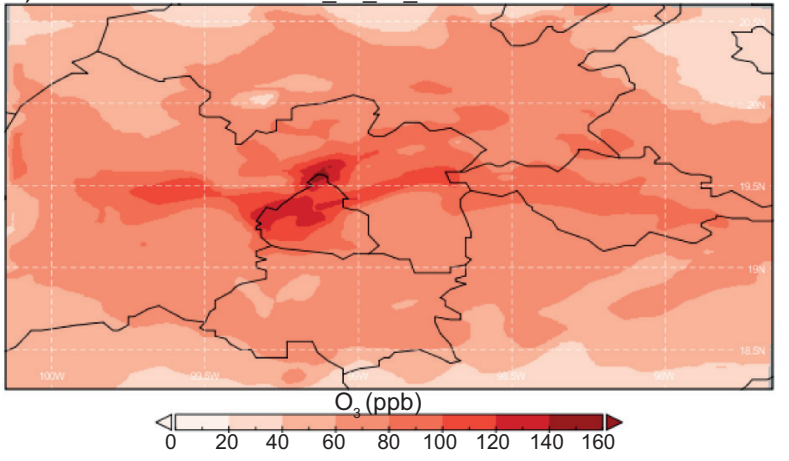

el NMB lo hace en el rango de 0 a $1.5 \%$ y el NME permanece siempre por debajo de $8 \%$. Los valores de ME y RMSE son aceptables para la escala de las variables. Para la estación HGM, que presenta los resultados más discretos en los estadísticos mostrados, el WRF-Chem subestima en más de $40 \mathrm{ppb}$ el valor máximo de concentración de $\mathrm{O}_{3}$ alcanzado el día 14 .

Finalmente, en la figura 23 se muestra la distribución de las concentraciones medias de $\mathrm{O}_{3}$ simuladas en el dominio de modelación a partir del INEM-2013 actualizado, para diferentes horarios del 15 de marzo de 2016. Se manifiesta la capacidad del WRF-Chem para modelar el comportamiento espacial de la variable, con máximos observados en zonas ubicadas dentro de la ZMVM o muy próximas a ésta, y una descripción adecuada de su ciclo diario con aumento de las concentraciones en horas de la tarde.

\section{CONCLUSIONES}

En este estudio se aplica el modelo DiETE al INEM-2013 para obtener una BDE útil para la modelación de la calidad del aire en el centro de México.

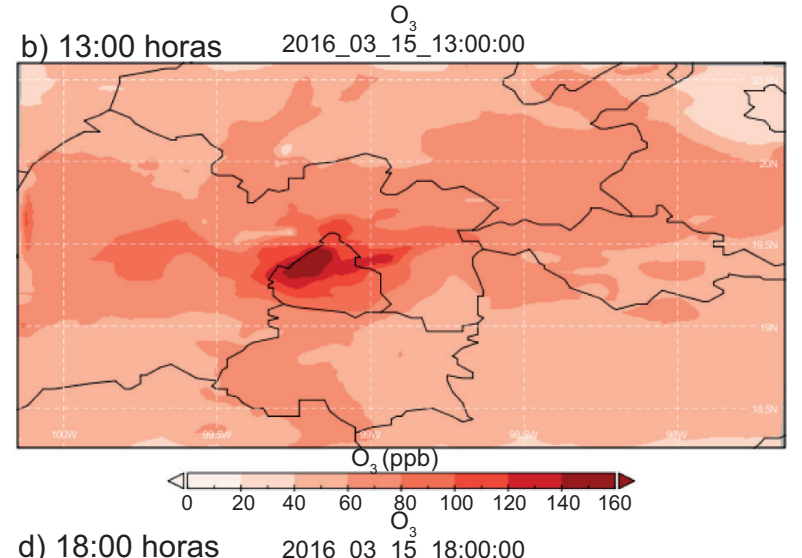

d) 18:00 horas 2016_03_15_18:00:00

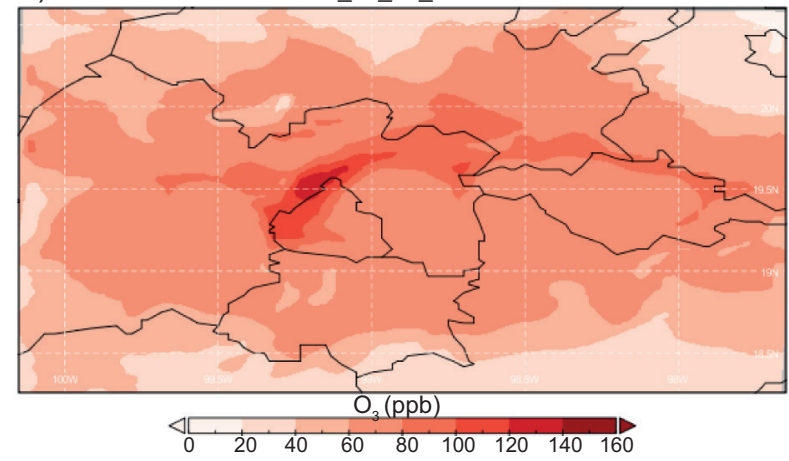

Fig. 23. Distribución espacial de las concentraciones de $\mathrm{O}_{3}$ (en ppb) obtenidas a partir del INEM-2013 actualizado (15 de marzo de 2016. (a) 08:00, (b) 13:00, (c) 16:00 y (d) 18:00 horas. 
Se realizan simulaciones numéricas con el modelo WRF-Chem en periodos de tiempo seleccionados, teniendo en cuenta la activación del PCAA en la ZMVM para 2016 y 2017.

Los resultados obtenidos se comparan con observaciones de estaciones de la RAMA disponibles para el caso de estudio de 2017 y se muestra que el INEM-2013 se puede actualizar para describir el comportamiento de los contaminantes que afectan el área de interés. Esto se sustenta en el análisis de las métricas estadísticas calculadas para cada variable y las series de tiempo que se obtienen para $\mathrm{O}_{3}$, en sus concentraciones medias y máximas. Los valores simulados difieren de los considerablemente observados, sobre todo en los registros extremos, con tendencia a subestimar las concentraciones de $\mathrm{CO}_{\text {y }} \mathrm{O}_{3}$ y sobrestimar $\mathrm{NO}_{2}, \mathrm{NO}$ y SO 2 , superando el $700 \%$ para $\mathrm{NO}_{2}$.

Tomando en cuenta las incertidumbres existentes durante el proceso de elaboración de un IE, lo cual puede conducir a errores en las simulaciones numéricas con MCA, se lleva a cabo un proceso de actualización del INEM-2013 a partir de una metodología basada en el cálculo de factores de escalamiento. De esta forma se obtiene un conjunto de estos factores para las emisiones de contaminantes criterio, con el fin de obtener una BDE actualizada con la cual los resultados del WRF-Chem muestren concentraciones de $\mathrm{O}_{3}$ similares a las observadas.

Se evalúan nuevamente las salidas del modelo y se comprueba que el empleo del INEM-2013 actualizado produce una mejoría considerable en el desempeño del WRF-Chem, según los valores de los estadísticos considerados. Las cifras de IOA y son siempre superiores a 0.65 para todos los contaminantes, llegando a 0.88 y 0.86 , respectivamente, en el caso del $\mathrm{O}_{3}$, el más importante en el área de interés. Precisamente el $\mathrm{O}_{3}$ presenta concentraciones similares a las observadas en cuanto a su distribución temporal (ciclo diario característico y valores medios y extremos consistentes con las mediciones) y en su comportamiento espacial (máximas concentraciones en puntos dentro de la ZMVM). Debe señalarse que el modelo mantiene la tendencia a subestimar las observaciones de $\mathrm{O}_{3}$, aunque solo ligeramente, con un $\mathrm{MB}=-3.89$ y NMB de sólo $-0.34 \%$.

Los factores de escalamiento obtenidos son validados mediante la evaluación de los resultados de las simulaciones numéricas realizadas para el caso de estudio de 2016, los cuales son nuevamente superiores al emplearse el INEM-2013 actualizado, incluso respecto al primer periodo. Los valores de IOA y $\rho$ sobrepasan 0.70 para todos los contaminantes, mejorando considerablemente los registros iniciales para $\mathrm{NO}_{2}$ y NO. En el caso del $\mathrm{O}_{3}$, ambas métricas superan 0.90 y se mantiene el buen desempeño del WRF-Chem en la simulación de sus características espacio-temporales, aunque persiste en subestimar las observaciones ligeramente, con $\mathrm{MB}=-3.97$ y NMB $=-0.48 \%$, comportamiento que se acrecienta en los picos máximos, con diferencias que superan $40 \mathrm{ppb}$.

También se presentan las métricas estadísticas para todas las variables y series de tiempo de $\mathrm{O}_{3}$ para un conjunto de estaciones de la RAMA, seleccionadas en cada caso de estudio, teniendo como denominador común el hecho de que la estación HGM presenta los resultados más discretos.

En cuanto a las variables meteorológicas evaluadas $\left(\mathrm{T}_{2}, \mathrm{HR}\right.$ y VV), el modelo presenta siempre un excelente rendimiento, ligeramente superior cuando se emplea el INEM-2013 actualizado.

En general, se demuestra que el INEM-2013 actualizado a partir de una metodología consistente, permite obtener una BDE lista para modelación de la calidad del aire con WRF-Chem en el centro de México, con altas correlaciones en los resultados, tanto en la descripción del comportamiento de variables meteorológicas como de contaminantes atmosféricos que afectan el área de interés, principalmente el $\mathrm{O}_{3}$.

Se recomienda utilizar la metodología propuesta para identificar los factores de escalamiento de forma estacional y también la obtención de éstos para la distribución espacial dentro del área de estudio, mediante el empleo de observaciones satelitales. Finalmente, este proceso puede aplicarse para contribuir a la identificación de la incertidumbre en los IE.

\section{AGRADECIMIENTOS}

Los autores agradecen el apoyo del Consejo Nacional de Ciencia y Tecnología (CONACYT) por la beca núm. 627876 (CVU) otorgada a José Alejandro Rodríguez Zas para realizar sus estudios de doctorado en Ciencias de la Tierra. Además, agradecen el tiempo de cómputo otorgado por la Universidad Nacional Autónoma de Mexico (UNAM) en la supercomputadora Miztli de la Dirección General de Cómputo y Tecnologías de Información y Comunicación (DGTIC) y en el clúster Ometéotl del Centro de ciencias de la Atmósfera (CCA).

\section{REFERENCIAS}

Arriaga-Colina J.L., West J.J., Sosa G., Escalona S.S., Orduñez R.M. y Cervantes A.D.M. (2004). Measurements of 
VOCs in Mexico City (1992-2001) and evaluation of VOCs and $\mathrm{CO}$ in the emissions inventory. Atmos. Environ. 38, 2523-2533. https://doi.org/10.1016/j. atmosenv.2004.01.033

Borrego C., Monteiro A., Ferreira J., Miranda A.I., Costa A.M., Carvalho A.C. y Lopes M. (2008). Procedures for estimation of modeling uncertainty in air quality assessment. Environ. Int. 34, 613-620. https://doi. org/10.1016/j.envint.2007.12.005

Brown M. (1993). Deduction of emission of source gases using an objective inversion algorithm and a chemical transport model. J. Geophys. Res. 98, 12639-12660. https://doi.org/10.1029/93JD01003

CAMe (2016). Se activa fase i de contingencia ambiental atmosférica por ozono en la Zona Metropolitana del Valle de México. Comunicado de prensa núm. 06/2016. Comisión Ambiental de la Megalópolis, Ciudad de México, 14 de marzo [en línea]. https://www.gob.mx/ comisionambiental/prensa/comunicado-de-prensa26192?idiom=es

CARB (1997). Public meeting to consider approval and verification of the California Emissions Inventory. California Air Resources Board, Sacramento, California.

Chang M.E., Hartley D.E., Cardelino C., Haas-Laursen D. y Chang W.L. (1997). On using inverse methods for resolving emissions with large spatial inhomogeneities. J. Geophys. Res. 102, 16023-16036. https:// doi.org/10.1029/97JD00964

Chang J.C. y Hanna S.R. (2004). Air quality model performance evaluation. Meteorol. Atmos. Phys. 87, 167-196. https://doi.org/10.1007/s00703-003-0070-7

Chen F. y Dudhia J. (2001). Coupling an advanced land surface-hydrology model with the Penn StateNCAR MM5 modeling system. Part I: Model description and implementation. Mon. Weather Rev. 129, 569-585. https://doi.org/10.1175/15200493(2001)129<0569:CAALSH >2.0.CO;2

De Foy B., Clappier A., Molina L.T. y Molina M.J. (2006). Distinct wind convergence patterns in the Mexico City basin due to the interaction of the gap winds with the synoptic flow. Atmos. Chem. Phys. 6, 1249-1265. https://doi.org/10.5194/acp-6-1249-2006

Dudhia J. (1989). Numerical study of convection observed during the Winter Monsoon Experiment using a mesoscale two-dimensional model. J. Atmos. Sci. 46, 3077-3107. https://doi.org/10.1175/15200469(1989)046<3077:NSOCOD >2.0.CO;2

Elbern H., Strunk A., Schmidt H. y Talagrand O. (2007). Emission rate and chemical state estimation by 4-dimensional variational inversion. Atmos. Chem. Phys. 7, 3749-3769. https://doi.org/10.5194/acp-7-37492007
Fast J.D., Gustafson W.I. Jr., Easter R.C., Zaveri R.A., Barnard J.C., Chapman E.G., Grell G.A. y Peckham S.E. (2006). Evolution of ozone, particulates, and aerosol direct radiative forcing in the vicinity of Houston using a fully coupled meteorology-chemistry-aerosol model. J. Geophys. Res. 111, D21305. https://doi. org/10.1029/2005JD006721

Forkel R., Werhahn J., Hansen A.B., McKeen S., Peckham S., Grell G. y Suppan P. (2012). Effect of aerosolradiation feedback on regional air quality - A case study with WRF/Chem. Atmos. Environ. 53, 202-211. https://doi.org/10.1016/j.atmosenv.2011.10.009

Fujita E.M., Croes B.E., Bennett C.L., Lawson D.R., Lurmann F.W. y Main H.H. (1992). Comparison of emission inventory and ambient concentration ratios of CO, NMOG and NOx in California's South Coast Air Basin. J. Air Waste Manage. Assoc. 42 (3), 264276. https://doi.org/10.1080/10473289.1992.10466989

Funk T.H., Chinkin L.R., Roberts P.T., Saeger M., Mulligan S., Figueroa V.H.P. y Yarbrough J. (2001). Compilation and evaluation of a Paso del Norte emission inventory. Sci. Total Environ. 276, 135-151. https:// doi.org/10.1016/s0048-9697(01)00776-8

García-Reynoso J.A, Mar-Morales B.E. y Ruiz-Suárez L.G. (2018). Modelo de distribución espacial, temporal y de especiación del Inventario de Emisiones de México (año base 2008) para su uso en modelización de calidad del aire (DiETE). Rev. Int. Contam. Ambie. 34 (4), 635-649. https://doi.org/10.20937/ RICA.2018.34.04.07

Grell G.A., Peckham S.E., Schmitz R., McKeen S.A., Frost G., Skamarock W.C. y Eder B. (2005). Fully coupled "online" chemistry within the WRF model. Atmos. Environ. 39, 6957-6975. https://doi.org/10.1016/j. atmosenv.2005.04.027

Goldberg D.E. (1989). Genetic algorithm in search, optimisation and machine learning. Addison-Wesley, Reading, MA, $415 \mathrm{pp}$.

Guenther A., Zimmerman P. y Wildermuth M. (1994). Natural volatile organic compound emission rate estimates for U.S. Woodland landscapes. Atmos. Environ. 28, 1197-1210. https://doi.org/10.1016/13522310(94)90297-6

Guenther A., Karl T., Harley P., Wiedinmyer C., Palmer P.I. y Geron, C. (2006). Estimates of global terrestrial isoprene emissions using MEGAN (Model of Emissions of Gases and Aerosols from Nature). Atmos. Chem. Phys. 6, 3181-3210. https://doi.org/10.5194/acp-6-3181-2006

Guilliland A.B., Appel K.W., Pinder R.W. y Dennis R.L. (2006). Seasonal $\mathrm{NH}_{3}$ emissions for the continental United States: inverse model estimation and evaluation. Atmos. Environ. 40, 4986-4998. https://doi. org/10.1016/j.atmosenv.2005.12.066 
Hakami A., Henze D.K., Seinfeld J.H., Chai T., Tang Y., Carmichael G.R. y Sandu A. (2005). Adjoint inverse modeling of black carbon during the Asian Pacific Regional Aerosol Characterization Experiment. J. Geophys. Res. 110, D14301. https://doi. org/10.1029/2004JD005671

Hao J., Tian H. y Lu Y. (2002). Emission inventories of NOx from commercial energy consumption in China, 1995-1998. Environ. Sci. Technol. 36, 552-560. https:// doi.org/10.1021/es015601k

Hernández J.O. (2006). Actualización del inventario de emisiones para el pronóstico de la calidad del aire en la Zona Metropolitana de la Ciudad de México. Tesis de Licenciatura. Facultad de Estudios Superiores Zaragoza, Universidad Nacional Autónoma de México. Ciudad de México, México, 86 pp.

Holland J.H. (1975). Adaptation in natural and artificial systems. The University of Michigan Press, Ann Arbor, MI, 183 pp.

Hong S.Y., Dudhia J. y Chen S.H. (2004). A revised approach to ice microphysical processes for the bulk parameterization of clouds and precipitation. Mon. Weather. Rev. 132, 103-120. https://doi. org/10.1175/1520-0493(2004)132<0103:ARATIM> 2.0.CO;2

Hong S.Y., Noh Y. y Dudhia J. (2006). A new vertical diffusion package with an explicit treatment of entrainment processes. Mon. Weather Rev. 134, 2318-2341. https:// doi.org/10.1175/MWR3199.1

Huang C., Chen C.H., Li L., Cheng Z., Wang H.L., Huang H.Y., Streets D.G., Wang Y.J., Zhang G.F. y Chen Y.R. (2011). Emission inventory of anthropogenic air pollutants and VOC species in the Yangtze River Delta region, China. Atmos. Chem. Phys. 11 (9), 4105-4120. https://doi.org/10.5194/acp-11-4105-201

INECC (2017). Preparación de un inventario de emisiones para modelación. Instituto Nacional de Ecología y Cambio Climático. Informe final. Ciudad de México, México, 35 pp. [en línea]. https://www.gob.mx/cms/ uploads/attachment/file/310811/Informe_INECC_ RP_A1-004 2017_Inventario_para_Modelaci_n_ $\mathrm{V} 3 . \mathrm{pdf}$

INECC (2018). Inventario Nacional de Emisiones de Gases y Compuestos de Efecto Invernadero 1990-2015. Instituto Nacional de Ecología y Cambio Climático. Resumen Informativo. Ciudad de México, México, 10 pp. [en línea]. https://cambioclimatico.gob.mx/sextacomunicacion/material/Inventario.pdf

IPCC (2000). Good practice guidance and uncertainty management in national greenhouse gas inventories. Panel Intergubernamental de Cambio Climático [en línea]. http://www.ipcc-nggip.iges.or.jp/public/gp/ english
Kain J.S. y Fritsch J.M. (1993). Convective parameterization for mesoscale models: the Kain-Fritsch scheme. En: The representation of cumulus convection in numerical models (Emanuel K.A. y Raymond D.J., Ed.). Amer. Meteor. Soc. 165-170. https://doi. org/10.1007/978-1-935704-13-3_16

Kain J.S. (2004). The Kain-Fritsch convective parameterization: an update. J. Appl. Meteorol. 43, 170-181. https://doi.org/10.1175/1520-0450(2004)0432.0.CO;2

Keenan T., Niinemets U., Sabate S., Gracia C. y Penuelas J. (2009). Process based inventory of isoprenoid emissions from European forests: Model comparisons, current knowledge and uncertainties. Atmos. Chem. Phys. 9 (12), 4053-4076. https://doi.org/10.5194/acp9-4053-2009

Kurokawa J., Yumimoto K., Uno I. y Ohara T. (2009). Adjoint inverse modeling of $\mathrm{NO}_{\mathrm{x}}$ emissions over Eastern China using satellite observations of $\mathrm{NO}_{2}$ vertical column densities. Atmos. Environ. 43 (11), 1878-1887. https://doi.org/10.1016/j.atmosenv.2008.12.030

Li M.J., Chen D.S., Cheng S.Y., Wang F., Li Y., Zhou Y. y Lang J.L. (2010). Optimizing emission inventory for chemical transport models by using genetic algorithm. Atmos. Environ. 44 (32), 3926-3934. https:// doi.org/10.1016/j.atmosenv.2010.07.010

Lumbreras J., García-Martós C., Mira J. y Borge R. (2009). Computation of uncertainty for atmospheric emission projections from key pollutant sources in Spain. Atmos. Environ. 43 (8), 1557-1564. https://doi.org/10.1016/j. atmosenv.2008.10.048

Lumbreras J., de Andrés J.M., Pérez J., Borge R., de la Paz D. y Rodríguez M.E. (2015). A methodology to estimate uncertainty for emission projections through sensitivity analysis. J. Air Waste Manage. Assoc. 65 (4), 384-394. https://doi.org/10.1080/10962247.201 4.996268

Mannschreck K., Klemp D., Kley D., Friedrich R., Kuhlwein J., Wickert B., Matuska P., Habram M. y Slemr F. (2002). Evaluation of an emission inventory by comparisons of modeled and measured emissions ratios of individual $\mathrm{HCs}, \mathrm{CO}$ and $\mathrm{NO}_{\mathrm{x}}$. Atmos. Environ. 36, 81-94. https://doi.org/10.1016/S13522310(02)00211-X

Mendoza-Domínguez A. y Russell A.G. (2001). Estimation of emission adjustments from the application of four-dimensional data assimilation to photochemical air quality modeling. Atmos. Environ. 35 (16), 28792894. https://doi.org/10.1016/S1352-2310(01)00084$\mathrm{X}$

Mendoza A. y García M.R. (2011). Modelación inversa aplicada al análisis del inventario de emisiones de la Zona Metropolitana de Guadalajara, México. Rev. Int. Contam. Ambie. 27 (3), 199-214. 
Miller C.A., Hidy G., Hales J., Kolb C.E., Werner A.S., Haneke B., Parrish D., Frey H.C., Rojas-Bracho L., Deslauriers M., Pennell B. y Mobley J.D. (2012). Air emission inventories in North America: A critical assessment. J. Air Waste Manage. Assoc. 56, 1115-29. https://doi.org/10.1080/10473289.2006. 10464540

Mlawer E.J., Taubman S.J., Brown P.D., Iacono M.J. y Clough S.A. (1997). Radiative transfer for inhomogeneous atmospheres: RRTM, a validated correlated-k model for the longwave. J. Geophys. Res. Atmos. 102, 16663-16682. https://doi. org/10.1029/97JD00237

Mulholland M. y Seinfeld J.H. (1995). Inverse air pollution modeling of urban scale carbon monoxide emissions. Atmos. Environ. 29, 497-516. https://doi. org/10.1016/1352-2310(94)00245-G

Napelenok S.L., Pinder R.W., Gilliland A.B. y Martin R.V. (2008). A method for evaluating spatially resolved $\mathrm{NO}_{\mathrm{x}}$ emissions using Kalman filter inversion, direct sensitivities and space-based $\mathrm{NO}_{2}$ observations. Atmos. Chem. Phys. 8, 5603-5614. https://doi.org/10.5194/ acp-8-5603-2008

Pielke R.A. (1984). Mesoscale meteorological modeling. Academic Press, London.

Raadgever G.T., Dieperink C., Driessen P.P.J., Smit A.A.H. y van Rijswick H.F.M.W. (2011). Uncertainty management strategies: Lessons from the regional implementation of the Water Framework Directive in the Netherlands. Environ. Sci. Policy. 14, 64-75. https://doi.org/10.1016/j.envsci.2010.11.001

Rew R.K. y Davis G.P. (1990). NetCDF: An interface for scientific data access. IEEE Comput. Graph. Appl. 10 (4), 76-82. https://doi.org/10.1109/38.56302

Saide P., Osses A., Gallardo L. y Osses M. (2009). Adjoin inverse modeling of a $\mathrm{CO}$ emission inventory at the city scale: Santiago de Chile's case. Atmos. Chem. Phys. Discuss. 9, 6325-6361. https://doi.org/10.5194/ acpd-9-6325-2009

Schultz C. (2008). Responding to scientific uncertainty in U.S. forest policy. Environ. Sci. Policy. 11, 253-71. https://doi.org/10.1016/j.envsci.2007.09.002

SEDEMA (2016). Aviso por el que se da a conocer el Programa para Contingencias Ambientales Atmosféricas en la Ciudad de México. Secretaría del Medio Ambiente del Gobierno de la Ciudad de México. Gaceta Oficial de la Ciudad de México No. 44, 6 de abril.

SEMARNAT (2002). Programa para Mejorar la Calidad del Aire de la Zona Metropolitana del Valle de México (ProAire) 2002-2010. Secretaría de Medio Ambiente y Recursos Naturales. Informe Final. Ciudad de México, México, 386 pp.

SEMARNAT (2015). Guía para la elaboración de los programas de gestión para mejorar la calidad del aire
(ProAire). Secretaría de Medio Ambiente y Recursos Naturales. Informe Final. Ciudad de México, México, 46 pp.

SEMARNAT (2017). Se activa fase I de contingencia ambiental atmosférica por ozono. Comisión Ambiental de la Megalópolis (CAMe). Secretaría de Medio Ambiente y Recursos Naturales. Comunicado de Prensa No. 47/2017. Ciudad de Mexico, 15 de mayo [en línea]. https:/www.gob.mx/semarnat/prensa/se-activa-fasei-de-contingencia-ambiental-atmosferica-por-ozono107342?idiom=es-MX

Sowden M., Cairncross E., Wilson G., Zunckel M., Kirillova E., Reddy V. y Hietkamp S. (2008). Developing a spatially and temporally resolved emission inventory for photochemical modeling in the City of Cape Town and assessing its uncertainty. Atmos. Environ. 42 (30), 7155-7164. https://doi.org/10.1016/j. atmosenv.2008.05.048

SSA (2014). Norma Oficial Mexicana NOM-020SSA1-2014. Salud ambiental. Valor límite permisible para la concentración de ozono $\left(\mathrm{O}_{3}\right)$ en el aire ambiente y criterios para su evaluación. Secretaría de Salud. Diario Oficial de la Federación, 19 de agosto.

Stephens S., Madronich F., Wu F., Olson J. B., Ramos R., Retama A. y Muñoz R. (2008). Weekly patters of Mexico City's surface concentrations of $\mathrm{CO}, \mathrm{NO}_{\mathrm{x}}$, $\mathrm{PM}_{10}$ and $\mathrm{O}_{3}$ during 1986-2007. Atmos. Chem. Phys. 8, 5313-5325. https://doi.org/10.5194/acp-85313-2008

Stockwell W., Middelton P., Chang J. y Xiaoyan T. (1990). The second-generation Regional Acid Deposition Model chemical mechanism for regional air quality modeling. J. Geophys. Res. Atmos. 95, 16343-18367. https://doi.org/10.1029/JD095iD10p16343

Streets D.G., Bond T.C., Carmichael G.R., Fernandes S.D., Fu Q., He D., Klimont Z., Nelson S.M., Tsai N.Y., Wang M.Q., Woo J.H. y Yarber K.F. (2003). The MICS-Asia Phase II emission inventory. En: The Sixth Workshop on the Transport of Air Pollutants in Asia (Model Intercomparison StudydMICS-Asia). International Institute for Applied Systems Analysis, Laxenburg, Austria.

Streit G.E. y Guzmán F. (1993). Mexico City Air Quality Research Initiative (MARI). WIT Transactions on Ecology and the Environment 1, 599-609.

Streit G.E. y Guzmán F. (1996). Mexico City air quality: Progress of an international collaborative project to define air quality management options. Atmos. Env. 30 (5), 723-733. https://doi.org/10.1016/13522310(95)00275-8

Tie X., Madronich S., Li G.H., Ying Z., Zhang R., García A., Lee-Taylor J. y Liu Y. (2007). Characteristics of chemical oxidants in Mexico City: A regional chemical 
dynamical model (WRF-Chem) study. Atmos. Environ. 41, 1989-2008. https://doi.org/10.1016/j. atmosenv.2006.10.053

Trejo V. (2017) Comparación de dos mecanismos químicos en la modelación de la calidad del aire en el Valle de México. Tesis de Licenciatura. Facultad de Química, Universidad Nacional Autónoma de México. Ciudad de México, México, 78 pp.

Turtós L., Capote G., Fonseca Y., Álvarez L., Sánchez M., Bezanilla A., Borrajero I., Meneses R. y Pire S. (2013). Assessment of the Weather Research and Forecasting model implementation in Cuba addressed to diagnostic air quality modeling. Atmos. Pollut. Res. 4 (1), 64-74. https://doi.org/10.5094/APR.2013.007

Watson J.G., Chow J.C. y Fujita E.M. (2001). Review of volatile organic compound source apportionment by chemical mass balance. Atmos. Environ. 35 (9), 15671584. https://doi.org/10.1016/S1352-2310(00)00461-1

West J.J, Zavala M.A., Molina L.T., Molina M.J., San Martini F., McRae G.J., Sosa-Iglesias G. y Arriaga-Colina J.L. (2004). Modeling ozone photochemistry and Evaluation of hydrocarbon emissions in the Mexico City metropolitan area. J. Geophys. Res. 109, D19312. https://doi.org/10.1029/2004JD004614.

Willmott C.J. (1981). On the validation of models. Phys. Geogr. 2, 184-194. https://doi.org/10.1080/02723646 .1981 .10642213
Willmott C.J., Ackleson S.G., Davis R.E., Feddema J.J., Klink K.M., Legates D.R., O'Donnell J. y Rowe C.M. (1985). Statistics for the evaluation of model performance. J. Geophys. Res. 90, 8995-9005. https://doi. org/10.1029/JC090iC05p08995

Ying Z., Shuiyuan C., Jianbing L., Jianlei L., Li L. y Dongsheng C. (2012). A new statistical modeling and optimization framework for establishing highresolution $\mathrm{PM}_{10}$ emission inventory - II. Integrated air quality simulation and optimization for performance improvement. Atmos. Environ. 60, 623-631. https:// doi.org/10.1016/j.atmosenv.2012.07.055

Yumimoto K. y Uno I. (2006). Adjoint inverse modeling of $\mathrm{CO}$ emissions over Eastern Asia using fourdimensional variational data assimilation. Atmos. Environ. 40, 6836-6845. https://doi.org/10.1016/j. atmosenv.2006.05.042

Zhang D. y Anthes R.A. (1982). A high-resolution model of the planetary boundary layer - Sensitivity tests and comparisons with SESAME-70 Data. J. Appl. Meteorol. 21, 1594-1609. https://doi.org/10.1175/15200450(1982)021<1594:AHRMOT>2.0.CO;2

Zhang Y., Dubey M.K., Olsen S.C., Zheng J. y Zhang R. (2009). Comparisons of WRF-Chem simulations in Mexico City with ground-based RAMA measurements during the 2006-MILAGRO. Atmos. Chem. Phys. 9 (11) 3777-3798. https://doi.org/10.5194/acp-9-3777-2009 